\title{
SOLVING VARIATIONAL INEQUALITIES INVOLVING NONEXPANSIVE TYPE MAPPINGS
}

\author{
N. C. WONG, D. R. SAHU AND J. C. YAO
}

\begin{abstract}
The purpose of this paper is to investigate the asymptotic behavior of algorithms for finding solutions for a certain class of variational inequalities $V I_{D}(C, I-f)$ involving nonexpansive type mappings in smooth Banach spaces. We study existence of solutions of variational inequalities $V I_{D}(C, I-f)$ when $D$ is the set of solutions of zeros of accretive operators or the set of fixed points of nonexpansive mappings or the set of fixed points of pseudocontractive mappings. Our convergence analysis covers proximal point algorithm for finding zeros accretive operators as well as functional Helpern algorithm for finding fixed points of nonexpansive mappings in Banach spaces. Our results improve a number of results concerned with viscosity approximation methods in the context of weakly contraction mappings.
\end{abstract}

\section{INTRODUCTION}

Let $H$ be a real Hilbert space with inner product $\langle.,$.$\rangle and norm \|$.$\| , respectively.$ Let $C$ be a nonempty closed convex subset of $H$ and $A: C \rightarrow H$ a nonlinear mapping. $A$ is said to be monotone if $\langle A x-A y, x-y\rangle \geq 0$ for all $x, y \in C$. A monotone operator $A$ is said to be maximal monotone if its graph is not properly contained in the graph of any other monotone operator on $H$.

The variational inequality $V I(C, A)$ is formulated as finding a point $z \in C$ such that

$$
\langle A z, z-v\rangle \geq 0 \text { for all } v \in C .
$$

The variational inequalities were initially studied by Stampachhia [10, 12] and ever since have been widely studied. Such a problem is connected with the convex minimization problem, the complementarity problem, the problem of finding a point $u \in H$ satisfying $0=A u$ and so on.

Existence and approximation of solutions are important aspects of study of variational inequalities. It is well known that if $A$ is Lipschitzian and strongly monotone, then for small $\mu>0$, the mapping $P_{C}(I-\mu A)$ is a contraction. In this case, the Banach contraction principle guarantees that $V I(C, A)$ has unique solution $x^{*}$ and

\footnotetext{
Key words and phrases. Pseudocontractive mapping, uniformly Gâteaux differentiable norm, variational inequality, weakly contraction

2000 Mathematics Subject Classification: 47H06, 47H10, 47H10, 47J20

The first author was partially supported by a grant from National Science Council. The second author wishes to acknowledge for support of Department of Science and Technology, India, made in the program year 2007-2008, Project No. SR/FTP/MS-04.

Corresponding author: J.C. Yao, Tel.: +886- 7- 5253816; fax: +886- 7- 5253809.
} 
the sequence of Picard iteration process, given by, $x_{n+1}=P_{C}(I-\mu A) x_{n}$, converges strongly to $x^{*}$, where $P_{C}$ is the metric projection from $H$ onto $C$.

Throughout this paper $X$ is a real Banach space. Let $C$ be a convex subset of a smooth Banach space $X, D$ a nonempty subset of $C$ and $f: C \rightarrow C$ a mapping. We consider the following variational inequality $V I_{D}(C, I-f)$ :

$$
\text { to find a } z \in D \text { such that }\langle(I-f) z, J(z-v)\rangle \leq 0 \text { for all } v \in D \text {, }
$$

where $J$ is the duality mapping from $X$ into $X^{*}$.

The set of solutions of the variational inequality $V I_{D}(C, I-f)$ is denoted by $\Omega_{D}(I-f)$, i.e.,

$$
\Omega_{D}(I-f)=\{u \in C:\langle(I-f) z, J(z-v)\rangle \leq 0 \text { for all } v \in D\} .
$$

We denote $F(T)$ the set of fixed points of mapping $T: C \rightarrow C$.

The viscosity approximation method is one of the important methods for existence and approximation solutions of variational inequalities $V I_{D}(C, I-f)$. The viscosity approximation method was first discussed by Moudafi [16] as below:

Theorem 1.1. (Theorem 2.1, Moudafi [16]) Let $C$ be a nonempty closed convex subset of a Hilbert space $H$. Let $T: C \rightarrow C$ be a nonexpansive mapping and $f: C \rightarrow C$ a contraction mapping with $F(T) \neq \emptyset$. Let $\left\{x_{n}\right\}$ be the sequence defined by the scheme:

$$
x_{n}=\frac{1}{1+\varepsilon_{n}} T x_{n}+\frac{\varepsilon_{n}}{1+\varepsilon_{n}} f x_{n} \text { for all } \in \mathbb{N},
$$

where $\varepsilon_{n}$ is a sequence $(0,1)$ with $\varepsilon_{n} \rightarrow 0$. Then $\left\{x_{n}\right\}$ converges strongly to the unique solution of the variational inequality:

to find a $\tilde{x} \in D$ such that $\langle(I-f) \tilde{x}, \tilde{x}-x\rangle \leq 0$ for all $x \in F(T)$,

where $I$ is the identity mapping. In other word, $\tilde{x}$ is the unique fixed point of $P_{F(T)} f$.

$\mathrm{Xu}$ 24] extended the viscosity approximation method proposed by Moudafi [16] for a nonexpansive mapping in a uniformly smooth Banach space. If $\Pi_{C}$ denotes the set of all contractions on $C$, then he proved the following theorem.

Theorem 1.2. (Theorem 4.1, $X u$ [24]) Let $C$ be a nonempty closed convex subset of a uniformly smooth Banach space $X, f \in \Pi_{C}$ and $T: C \rightarrow C$ a nonexpansive mapping with $F(T) \neq \emptyset$. Then the path $\left\{x_{t}: t \in(0,1)\right\}$ defined by

$$
x_{t}=t f x_{t}+(1-t) T x_{t}
$$

converges strongly to a point in $F(T)$. If we define $Q: \Pi_{C} \rightarrow F(T)$ by

$$
Q(f)=\lim _{t \rightarrow 0^{+}} x_{t}, \quad f \in \Pi_{C},
$$

then $Q(f)$ solves the variational inequality:

$$
\langle(I-f) Q(f), J(Q(f)-v)\rangle \leq 0, \quad f \in \Pi_{C} \text { and } v \in F(T) .
$$

There are already several viscosity-like methods in Hilbert and Banach spaces, and the research is intensively continued which are very useful for approximating to the common element of $F(T)$ and $\Omega_{D}(I-f)$, when $f$ is a contraction mapping and $T$ is a nonexpansive mapping. In such viscosity methods, the contraction mapping 
$f$ not only guarantees the existence of approximating curve $\left\{x_{t}\right\}$ defined by (1.1), but it also ensures the strong convergence of both $\left\{x_{t}\right\}$ and $\left\{x_{n}\right\}$ to an element of $F(T) \cap \Omega_{D}(I-f)$, where $\left\{x_{n}\right\}$ is a sequence in $C$ generated by the functional Halpern iteration process:

$$
x_{n+1}=\alpha_{n} f x_{n}+\left(1-\alpha_{n}\right) T x_{n}, \quad n \in \mathbb{N},
$$

where $\left\{\alpha_{n}\right\}$ is a sequence in $[0,1]$ satisfying appropriate conditions. When $f x=u$ for all $x \in C$, then $f$ is a contraction with the Lipschitz constant 0 and $(1.2)$ reduces to $x_{n+1}=\alpha_{n} u+\left(1-\alpha_{n}\right) T x_{n}$ which was first studied by Halpern [7].

At this stage, the following natural question arises:

Question 1.3. Let $C$ be a nonempty closed convex subset of a smooth Banach space $X, D$ a nonempty closed convex subset of $C$ and $f: C \rightarrow C$ a nonexpansive mapping. Under what conditions $\Omega_{D}(I-f) \neq \emptyset$ ?

On the other hand, a variety of problems, including convex programming and variational inequalities, can be formulated as finding of zeros of maximal monotone operators. Therefore, one of the most interesting and important problems in the theory of maximal monotone operators is to find an efficient iterative algorithm to compute approximately zeroes of maximal monotone operators. One method for solving zeros of maximal monotone operators is proximal point algorithm. Let $T$ be a maximal monotone operator in a Hilbert space $H$. The proximal point algorithm generates, for starting $x_{1} \in H$, a sequence $\left\{x_{n}\right\}$ in $H$ by

$$
x_{n+1}=\left(I+r_{n} T\right)^{-1} x_{n} \text { for all } n \in \mathbb{N},
$$

where $\left\{r_{n}\right\}$ is a sequence in $(0, \infty)$. Note that $(1.3)$ is equivalent to

$$
0 \in \frac{1}{r_{n}}\left(x_{n+1}-x_{n}\right)+T x_{n+1} \text { for all } \in \mathbb{N} .
$$

This was first introduced by Martinet [15]. If $f: H \rightarrow(\infty, \infty]$ is a proper lower semicontinuous convex function, then the algorithm reduces to

$$
x_{n+1}=\arg \min _{y \in H}\left\{f(y)+\frac{1}{2 r_{n}}\left\|x_{n}-y\right\|^{2}\right\} \text { for all } n \in \mathbb{N} .
$$

Rockafellar 20] studied the proximal point algorithm in the framework of Hilbert space and he proved the following:

Theorem 1.4. Let $H$ be a Hilbert space, $A \subset H \times H$ a maximal monotone operator and $J_{r}=(I+r A)^{-1}$ for all $r>0$. Let $\left\{x_{n}\right\}$ be a sequence in $H$ defined by $x_{1}=x \in H$ and

$$
x_{n+1}=J_{r_{n}} x_{n} \text { for all } n \in \mathbb{N},
$$

where $\left\{r_{n}\right\}$ is a sequence in $(0, \infty)$ such that $\liminf _{n \rightarrow \infty} r_{n}>0$. If $A^{-1} 0 \neq \emptyset$, then the sequence $\left\{x_{n}\right\}$ converges weakly to an element of $A^{-1} 0$.

Gulär 4] constructed a counterexample showing that the sequence generated by (1.4) does not converge strongly, in general. This brings us a natural question how to modify the proximal point algorithm so that strongly convergent sequence is guaranteed. Recently, Benavides, Acedo and Xu [2, Kamimura and Takahashi [8], Kim and Xu 9], Mainge [13, Nakajo [17] and Solodov and Svaiter 21] modified proximal point algorithm to generate strongly convergent sequences. Now our concern is the following: 
Question 1.5. Is it possible to modify the proximal point algorithm by noncontraction viscosity method so that it can generate a strongly convergent sequence?

It is our purpose in this paper to give affirmative answers of Questions 1.3 and 1.5 . In Section 2, we will recall the useful definitions and lemmas. Section 3 is devoted to deal with the problem of existence of common element of $D$ and $\Omega_{D}(I-f)$ when $f$ is a weakly contraction. Section 4 is focused on existence of solutions of variational inequalities $V I_{D}(C, I-f)$, under the hypothesis of nonexpansiveness or pseudocontractivity of the mapping $f$. The results of this section are of interest in their own right in the constructive fixed point theory. Section 5 focuses on iterative algorithms for finding solutions of variational inequalities $V I_{D}(C, I-f)$ when $f$ is a weakly contraction or nonexpansive mapping. Using the established results, we consider the problem of finding a common fixed point of finitely many nonexpansive mappings and the problem of finding a common zero of finitely many accretive operators in Section 6.

\section{Preliminaries}

Let $C$ be a nonempty subset of a Banach space $X$ and $T: C \rightarrow C$ a mapping. $\mathrm{T}$ is called a Lipschitzian mapping if there exists a constant $L>0$ such that $\|T x-T y\| \leq L\|x-y\|$ for all $x, y \in C$ and $L$ is called Lipschitz constant of $T$. A Lipschitzian mapping with Lipschitz constant $L$ is said to be a contraction if $L \in[0,1)$ and a nonexpansive if $L=1$. $T$ is called pseudocontractive if there exists $j(x-y) \in J(x-y)$ such that

$$
\langle T x-T y, j(x-y)\rangle \leq\|x-y\|^{2} \text { for all } x, y \in C,
$$

where $J: X \rightarrow 2^{X^{*}}$ is the normalized duality mapping which is defined by

$$
J(u)=\left\{j \in X^{*}:\langle u, j\rangle=\|u\|^{2},\|j\| .=\|u\|\right\} .
$$

An operator $T$ with domain $D(T)$ and range $R(T)$ in a Banach space $\mathrm{X}$ is said to be a weakly contraction if

$$
\|T x-T y\| \leq\|x-y\|-\psi(\|x-y\|) \text { for all } x, y \in C,
$$

where $\psi:[0, \infty) \rightarrow[0, \infty)$ is a continuous and nondecreasing function such that $\psi(0)=0, \psi(t)>0$ for $t>0$ and $\lim _{t \rightarrow \infty} \psi(t)=\infty$.

Remark 2.1. (1) If $\psi(t)=k t$ for all $t \geq 0$, where $k \in(0,1)$, then $T$ is a contraction with Lipschitz constant $1-k$.

(2) If domain $D(T)$ of $T$ is bounded, then the hypothesis $\lim _{t \rightarrow \infty} \psi(t)=\infty$ is not necessary.

The concept of weakly contraction mappings was introduced by Alber and GuerreDelabriere [1] in Hilbert space in 1997. They proved that every weakly contraction mapping has a unique fixed point in a Hilbert space. In 2001, Rhoades [19] proved the following very interesting fixed point theorem which is one of generalizations of weakly contraction principle of Alber and Guerre-Delabriere [1 in metric space setting.

Theorem 2.2. (Theorem 1, Rhoades [19]) Let $(X, d)$ be a complete metric space and $f: X \rightarrow X$ a weakly contraction mapping. Then $f$ has a unique fixed point. 
Theorem 2.3. (Theorem 2, Rhoades [19]) Let C a nonempty closed convex subset of a Banach space $X$ and $f: C \rightarrow C$ a weakly contraction mapping with the function $\psi$. Then the sequence $\left\{x_{n}\right\}$ degenerated by the algorithm:

$$
x_{n+1}=f x_{n} \text { for all } \in \mathbb{N} \text {, }
$$

converges strongly to $p$, with the following error estimate:

$$
\left\|x_{n}-p\right\| \leq \Phi^{-1}\left(\Phi\left(\left\|x_{1}-p\right\|\right)\right)-(n-1)
$$

where $\Phi$ is defined by antiderivative

$$
\Phi(t)=\int \frac{d t}{\psi(t)}, \quad \Phi(0)=0
$$

and $\Phi^{-1}$ is the inverse of $\Phi$.

Recall that an operator $A$ with domain $D(A)$ and range $R(A)$ in a Banach space $X$ is said to be accretive if, for each $x_{i} \in D(A)$ and $y_{i} \in A x_{i}(i=1,2)$, there is $j \in J\left(x_{1}-x_{2}\right)$ such that $\left\langle y_{1}-y_{2}, j\right\rangle \geq 0$, where $J$ is the duality mapping from $X$ to the dual space $X^{*}$. An accretive operator $A$ is said to satisfy the range condition if $\overline{D(A)} \subset R(1+\lambda A)$ for all $\lambda>0$. If $A$ is accretive, then we can define, for each $\lambda>0$, a nonexpansive single-valued mapping $J_{\lambda}: R(1+\lambda A) \rightarrow D(A)$ by $J_{\lambda}=(I+\lambda A)^{-1}$. It is called the resolvent of $A$. It is well known that for an accretive operator $A$ which satisfies the range condition, $A^{-1}(0)=F\left(J_{\lambda}\right)$ for all $\lambda>0$. We also define the Yosida approximation $A_{r}$ by $A_{r}=\left(I-J_{r}\right) / r$. We know that $A_{r} x \in A J_{r} x$ for all $x \in R(I+r A)$ and $\left\|A_{r} x\right\| \leq|A x|=\inf \{\|y\|: y \in A x\}$ for all $x \in D(A) \cap R(I+r A)$. An accretive operator $A$ is said to be $m$-accretive if $R(I+r A)=X$ for all $r>0$.

A continuous mapping $T$ with domain $D(T)$ and range $R(T)$ in a Banach space $X$ is said to be demicompact at 0 if for any bounded sequence $\left\{y_{n}\right\}$ in $D(T)$ such that $y_{n}-T y_{n} \rightarrow 0$ as $n \rightarrow \infty$, there exists a subsequence $\left\{y_{n_{k}}\right\}$ of $\left\{y_{n}\right\}$ and $y \in D(T)$ such that $y_{n_{k}} \rightarrow y$ as $k \rightarrow \infty$.

A closed convex subset $C$ of a Banach space $X$ is said to have normal structure if for each closed convex bounded subset $D$ of $C$ which contains at least two points, there exists an element $x$ of $D$ which is not a diametral point of $D$, i.e.,

$$
\sup \{\|x-y\|: y \in D\}<\operatorname{diam}(D),
$$

where $\operatorname{diam}(D)$ is the diameter of $D$.

The following theorem related to the existence of fixed points of nonexpansive mappings was proved in Kirk [1].

Theorem 2.4. (Kirk's fixed point theorem [11]). Let $X$ be a reflexive Banach space and let $C$ be a nonempty closed convex bounded subset of $X$ which has normal structure. Let $T$ be a nonexpansive mapping from $C$ into itself. Then $F(T)$ is nonempty.

Recall that a Banach space $X$ is said to be smooth provided the limit

$$
\lim _{t \rightarrow 0^{+}} \frac{\|x+t y\|-\|x\|}{t}
$$

exists for each $x$ and $y$ in $S=\{x \in X:\|x\|=1\}$. In this case, the norm of $X$ is said to be Gâteaux differentiable. It is said to be uniformly Gâteaux differentiable 
if for each $y \in S$, this limit is attained uniformly for $x \in S$. It is well known that $X$ is smooth if and only if any duality mapping on $X$ is single-valued. Also if $X$ has a uniformly Gâteaux differentiable norm, then the duality mapping is norm-to-weak* uniformly continuous on bounded sets. It is also well known that every uniformly smooth space (e.g., $L_{p}$ space, $1<p<\infty$ ) has uniformly Gâteaux differentiable norm (see e.g., [3]).

Let $C$ be a convex subset of a Banach space $X, D$ a nonempty subset of $C$, and $P$ a retraction from $C$ onto $D$, that is, $P x=x$ for each $x \in D$. A retraction $P$ is said to be sunny if $P(P x+t(x-P x))=P x$ for each $x \in C$ and $t \geq 0$ with $P x+t(x-P x) \in C$. If the sunny retraction $P$ is also nonexpansive, then $D$ is said to be a sunny nonexpansive retract of $C$. The sunny nonexpansive retraction $Q_{D}$ from $C$ onto $D$ is unique if $X$ is smooth. The following lemmas will be needed in the sequel.

Lemma 2.5. (Lemma 13.1, Goebel and Reich [5]) Let $C$ be a convex subset of a smooth Banach space $X, D$ a nonempty subset of $C$ and $Q_{D}$ a retraction from $C$ onto $D$. Then the following are equivalent:

(a) $Q_{D}$ is a sunny and nonexpansive.

(b) $\left\langle x-Q_{D} x, J\left(z-Q_{D} x\right)\right\rangle \leq 0$ for all $x \in C, z \in D$.

(c) $\left\langle x-y, J\left(Q_{D} x-Q_{D} y\right)\right\rangle \geq\left\|Q_{D} x-Q_{D} y\right\|^{2}$ for all $x, y \in C$.

Lemma 2.6. (Cioranescu [3], p.85) Let $C$ be a nonempty closed convex subset of a reflexive strictly convex Banach space $X$. Then there exists a unique point $x \in C$ such that $\|x\|=\inf \{\|z\|: z \in C\}$.

Lemma 2.7. (Proposition 5.3, Goebel and Reich [5]) Let $C$ be a nonempty closed convex subset of a strictly convex Banach space $X$ and $T: C \rightarrow C$ a nonexpansive mapping with $F(T) \neq \emptyset$. Then $F(T)$ is closed and convex.

Lemma 2.8. Let $C$ be a nonempty closed convex subset of a reflexive strictly convex Banach space $X$ and $T: C \rightarrow C$ a nonexpansive mapping with $F(T) \neq \emptyset$. Then there exists a unique point $v \in F(T)$ such that $\|v\|=\inf \{\|z\|: z \in F(T)\}$.

Proof. It follows from Lemmas 2.6 and 2.7

Lemma 2.9. (Alber and Guerre-Delabriere [1]) Let $\left\{\alpha_{n}\right\}$ and $\left\{\beta_{n}\right\}$ be two sequences of nonnegative real numbers such that $\lim _{n \rightarrow \infty} \frac{\beta_{n}}{\alpha_{n}}=0$ and $\sum_{n=1}^{\infty} \alpha_{n}=\infty$. Let $\left\{\lambda_{n}\right\}$ be a sequence of nonnegative real numbers satisfying the recursive inequality:

$$
\lambda_{n+1} \leq \lambda_{n}-\alpha_{n} \phi\left(\lambda_{n}\right)+\beta_{n} \text { for all } n \in \mathbb{N}
$$

where $\phi:[0, \infty) \rightarrow[0, \infty)$ is a continuous and nondecreasing function such that $\phi(0)=0$ and $\phi(t)>0$ for $t>0$. Then $\left\{\lambda_{n}\right\}$ converges to zero.

Lemma 2.10. (Lemma 1, Ha and Jung [6]) Let $X$ be a Banach space with a uniformly Gâteaux differentiable norm, $C$ a nonempty closed convex subset of $X$ and $\left\{x_{n}\right\}$ a bounded sequence in $X$. Let LIM be a Banach limit and $y \in C$. Then

$$
L I M_{n}\left\|x_{n}-y\right\|^{2}=\min _{z \in C} L I M_{n}\left\|x_{n}-z\right\|^{2}
$$

if and only if

$$
L I M_{n}\left\langle x-y, J\left(x_{n}-y\right)\right\rangle \leq 0 \text { for all } x \in C .
$$


Lemma 2.11. Let $X$ be a reflexive Banach space with a uniformly Gâteaux differentiable norm, $C$ a nonempty closed convex subset of $X$ and $T: C \rightarrow C$ a nonexpansive mapping. Let $L I M$ be a Banach limit and $\left\{z_{n}\right\}$ a bounded sequence in $C$ such that $z_{n}-T z_{n} \rightarrow 0$ as $n \rightarrow \infty$. Let

$$
M:=\left\{y \in C: L I M_{n}\left\|z_{n}-y\right\|^{2}=\inf _{x \in C} L I M_{n}\left\|z_{n}-x\right\|^{2}\right\} .
$$

Suppose that every closed convex bounded subset of $C$ has fixed point property for nonexpansive self-mappings. Then we have the following:

(a) There exists a point $u$ in $M$ such that $u=T u$.

(b) If $f: C \rightarrow C$ is a weakly contraction mapping with the function $\psi$ and $\left\langle z_{n}-f z_{n}, J\left(z_{n}-u\right)\right\rangle \leq 0$ for all $n \in \mathbb{N}$, then there exists a subsequence $\left\{z_{n_{i}}\right\}$ of $\left\{z_{n}\right\}$ such that $\left\{z_{n_{i}}\right\}$ converges strongly to $u$.

Proof. (a) Define the function $\varphi: C \rightarrow \mathbb{R}$ by $\varphi(x):=L I M_{n}\left\|z_{n}-x\right\|^{2}, x \in C$. Since $X$ is reflexive, $\varphi(x) \rightarrow \infty$ as $\|x\| \rightarrow \infty$, and $\varphi$ is continuous convex function, we have that the set

$$
M:=\left\{y \in C: \varphi(y)=\inf _{x \in C} \varphi(x)\right\},
$$

which is nonempty closed convex and bounded. Furthermore, $M$ is invariant under $T$. In fact, for each $y \in M$, we have

$$
\begin{aligned}
\varphi(T y) & =L I M_{n}\left\|z_{n}-T y\right\|^{2} \\
& \leq L I M_{n}\left\|T z_{n}-T y\right\|^{2} \\
& \leq L I M_{n}\left\|z_{n}-y\right\|^{2}=\varphi(y) .
\end{aligned}
$$

So, by the hypothesis, there exists a fixed point $u$ of $T$ in $M$.

(b) By Lemma 2.10, we have

$$
L I M_{n}\left\langle z, J\left(z_{n}-u\right)\right\rangle \leq 0 \text { for all } z \in C .
$$

In particular,

$$
L I M_{n}\left\langle f u-u, J\left(z_{n}-u\right)\right\rangle \leq 0 .
$$

Since $f$ is a weakly contraction, we have

$$
\begin{aligned}
\left\|z_{n}-u\right\|^{2}= & \left\langle z_{n}-f z_{n}, J\left(z_{n}-u\right)\right\rangle+\left\langle f z_{n}-f u, J\left(z_{n}-u\right)\right\rangle+\left\langle f u-u, J\left(z_{n}-u\right)\right\rangle \\
\leq & \left\langle z_{n}-f z_{n}, J\left(z_{n}-u\right)\right\rangle+\left\|f z_{n}-f u\right\|\left\|z_{n}-u\right\|+\left\langle f u-u, J\left(z_{n}-u\right)\right\rangle \\
\leq & \left\langle z_{n}-f z_{n}, J\left(z_{n}-u\right)\right\rangle+\left[\left\|z_{n}-u\right\|-\psi\left(\left\|z_{n}-u\right\|\right)\right]\left\|z_{n}-u\right\| \\
& +\left\langle f u-u, J\left(z_{n}-u\right)\right\rangle .
\end{aligned}
$$

Since $\left\langle z_{n}-f z_{n}, J\left(z_{n}-u\right)\right\rangle \leq 0$ for all $n \in \mathbb{N}$, it follows that

$$
\begin{aligned}
\psi\left(\left\|z_{n}-u\right\|\right)\left\|z_{n}-u\right\| & \leq\left\langle z_{n}-f z_{n}, J\left(z_{n}-u\right)\right\rangle+\left\langle f u-u, J\left(z_{n}-u\right)\right\rangle \\
& \leq\left\langle f u-u, J\left(z_{n}-u\right)\right\rangle .
\end{aligned}
$$

From $(2.2)$, we obtain

$$
L I M_{n} \psi\left(\left\|z_{n}-u\right\|\right)\left\|z_{n}-u\right\| \leq 0 .
$$

Therefore, there exists a subsequence $\left\{z_{n_{i}}\right\}$ of $\left\{z_{n}\right\}$ such that $z_{n_{i}} \rightarrow u$. 
Lemma 2.12. Let $X$ be a Banach space with a uniformly Gâteaux differentiable norm, $C$ a nonempty closed convex subset of $X, f: C \rightarrow C$ a continuous mapping, $T: C \rightarrow C$ a nonexpansive mapping and $\left\{x_{n}\right\}$ a bounded sequence in $C$ such that $\lim _{n \rightarrow \infty}\left\|x_{n}-T x_{n}\right\|=0$. Suppose $\left\{z_{t}\right\}$ is a path in $C$ defined by $z_{t}=t f z_{t}+(1-$ $t) T z_{t}, t \in(0,1)$ such that $z_{t} \rightarrow z$ as $t \rightarrow 0^{+}$. Then

$$
\limsup _{n \rightarrow \infty}\left\langle f z-z, J\left(x_{n}-z\right)\right\rangle \leq 0 .
$$

Proof. Since

$$
z_{t}-x_{n}=t\left(f z_{t}-x_{n}\right)+(1-t)\left(T z_{t}-x_{n}\right)
$$

we see that

$$
\begin{aligned}
\left\|z_{t}-x_{n}\right\|^{2}= & t\left\langle f z_{t}-x_{n}, J\left(z_{t}-x_{n}\right)\right\rangle+(1-t)\left\langle T z_{t}-x_{n}, J\left(z_{t}-x_{n}\right)\right\rangle \\
\leq & t\left[\left\langle f z_{t}-z_{t}, J\left(z_{t}-x_{n}\right)\right\rangle+\left\|z_{t}-x_{n}\right\|^{2}\right] \\
& +(1-t)\left[\left\langle T z_{t}-T x_{n}, J\left(z_{t}-x_{n}\right)\right\rangle+\left\|x_{n}-T x_{n}\right\|\left\|z_{t}-x_{n}\right\|\right] \\
\leq & t\left[\left\langle f z_{t}-z_{t}, J\left(z_{t}-x_{n}\right)\right\rangle+\left\|z_{t}-x_{n}\right\|^{2}\right] \\
& +(1-t)\left[\left\|z_{t}-x_{n}\right\|^{2}+\left\|x_{n}-T x_{n}\right\|\left\|z_{t}-x_{n}\right\|\right] .
\end{aligned}
$$

By boundedness of $\left\{x_{n}\right\}$ and $\left\{z_{t}\right\}$, we have

$$
\begin{aligned}
\left\langle f z_{t}-z_{t}, J\left(x_{n}-z_{t}\right)\right\rangle & \leq \frac{1}{t}\left\|x_{n}-T x_{n}\right\|\left\|z_{t}-x_{n}\right\| \\
& \leq \frac{1}{t}\left\|x_{n}-T x_{n}\right\| K_{1}
\end{aligned}
$$

for some $K_{1}>0$. Since $x_{n}-T x_{n} \rightarrow 0$ as $n \rightarrow \infty$, it infers that

$$
\limsup _{n \rightarrow \infty}\left\langle f z_{t}-z_{t}, J\left(x_{n}-z_{t}\right)\right\rangle \leq 0 .
$$

Further, since $z_{t} \rightarrow z$ as $t \rightarrow 0^{+}$, the set $\left\{z_{t}-x_{n}\right\}$ is bounded and the duality mapping $J$ is norm-to-weak ${ }^{*}$ uniformly continuous on bounded subsets of $X$, it follows that

$$
\begin{aligned}
\mid\langle f z- & \left.z, J\left(x_{n}-z\right)\right\rangle-\left\langle f z_{t}-z_{t}, J\left(x_{n}-z_{t}\right)\right\rangle \mid \\
= & \left|\left\langle f z-z, J\left(x_{n}-z\right)-J\left(x_{n}-z_{t}\right)\right\rangle+\left\langle f z-z-\left(f z_{t}-z_{t}\right), J\left(x_{n}-z_{t}\right)\right\rangle\right| \\
\leq & \left|\left\langle f z-z, J\left(x_{n}-z\right)-J\left(x_{n}-z_{t}\right)\right\rangle\right| \\
& +\left\|f z-z-\left(f z_{t}-z_{t}\right)\right\|\left\|x_{n}-z_{t}\right\| \rightarrow 0 \text { as } t \rightarrow 0^{+} .
\end{aligned}
$$

Let $\varepsilon>0$. Then there exists $\delta>0$ such that

$$
\left\langle f z-z, J\left(x_{n}-z\right)\right\rangle<\left\langle f z_{t}-z_{t}, J\left(x_{n}-z_{t}\right)\right\rangle+\varepsilon \text { for all } n \in \mathbb{N} \text { and } t \in(0, \delta) .
$$

Using 2.3), we get

$$
\begin{aligned}
\limsup _{n \rightarrow \infty}\left\langle f z-z, J\left(x_{n}-z\right)\right\rangle & \leq \limsup _{n \rightarrow \infty}\left\langle f z_{t}-z_{t}, J\left(x_{n}-z_{t}\right)\right\rangle+\varepsilon \\
& \leq \varepsilon
\end{aligned}
$$

Since $\varepsilon$ is arbitrary, we obtain that

$$
\limsup _{n \rightarrow \infty}\left\langle f z-z, J\left(x_{n}-z\right)\right\rangle \leq 0 .
$$




\section{VARIATIONAL INEQUALITIES INVOLVING WEAKLY CONTRACTION MAPPINGS}

We begin with existence of solutions of $V I_{D}(C, I-f)$ in a reflexive smooth Banach space when $f$ is a weakly contraction and $D=A^{-1} 0$.

Theorem 3.1. Let $X$ be a reflexive Banach space with a uniformly Gâteaux differentiable norm, $C$ a nonempty closed convex subset of $X, f: C \rightarrow C$ a weakly contraction mapping with the function $\psi$ and $A \subset X \times X$ an accretive operator with resolvent $J_{t}$ for $t>0$ such that

$$
\overline{D(A)} \subset C \subset \bigcap_{t>0} R(I+t A)
$$

and

$$
E=\left\{x \in C: x=J_{\rho} f x \text { for some } \rho>0\right\} \text { and } f(E) \text { are bounded. }
$$

Suppose that every closed convex bounded subset of $C$ has fixed point property for nonexpansive self-mappings. Then we have the following:

(a) for each $t>0, J_{t} f$ has a unique fixed point $z_{t} \in C$;

(b) $\left\{z_{t}\right\}$ converges strongly to $u \in A^{-1} 0$ as $t \rightarrow \infty$;

(c) $u$ is the unique solution of the variational inequality:

$$
\text { find } \tilde{x} \in A^{-1} 0 \text { such that }\langle(I-f) \tilde{x}, J(\tilde{x}-v)\rangle \leq 0 \text { for all } v \in A^{-1} 0 \text {. }
$$

Proof. (a) For each $t>0$, the mapping $T_{t}^{f}: C \rightarrow C$ defined by

$$
T_{t}^{f} x=J_{t} f x, x \in C
$$

is a weakly contraction mapping with the function $\psi$. Indeed, for $x, y \in C$, we have

$$
\left\|T_{t}^{f} x-T_{t}^{f} y\right\|=\left\|J_{t} f x-J_{t} f y\right\| \leq\|f x-f y\| \leq\|x-y\|-\psi(\|x-y\|) .
$$

By Theorem 2.2 there exists a unique fixed point $z_{t} \in C$ of $T_{t}^{f}$ and hence

$$
z_{t}=J_{t} f z_{t}
$$

(b) By 3.2 , we see that $\left\{z_{t}\right\}$ is bounded. Since $f(E)$ is bounded, it follows that $\left\{\left\|z_{t}-f z_{t}\right\|\right\}$ is bounded. Hence, for $r>0$, we have

$$
\begin{aligned}
\left\|z_{t}-J_{r} z_{t}\right\| & =r\left\|A_{r} z_{t}\right\| \\
& \leq r\left|A z_{t}\right|=r\left|A J_{t} f z_{t}\right| \\
& \leq r\left\|A_{t} f z_{t}\right\|=\frac{r}{t}\left\|f z_{t}-J_{t} f z_{t}\right\|=\frac{r}{t}\left\|z_{t}-f z_{t}\right\| \\
& \leq \frac{r}{t} K_{2}
\end{aligned}
$$

for some $K_{2}>0$. We may assume that $\left\{t_{n}\right\}$ is a sequence in $(0, \infty)$ such that $\lim _{n \rightarrow \infty} t_{n}=\infty$ and $\left\{z_{t_{n}}\right\}$ is bounded. Set $z_{n}:=z_{t_{n}}$. For $r>0$, we see from 3.4 $n \rightarrow \infty$
that

$$
\left\|z_{n}-J_{r} z_{n}\right\| \leq \frac{r}{t_{n}} K_{2} \rightarrow 0 \text { as } n \rightarrow \infty .
$$

By Lemma 2.11 (a), there exists an element $u \in M \cap F\left(J_{r}\right)$. Thus, $A^{-1} 0 \neq \emptyset$. Since $A$ is accretive, we obtain from 3.3 that

$$
\frac{1}{t}\left(f z_{t}-z_{t}\right)=\frac{1}{t}\left(I-J_{t}\right) f z_{t}=A_{t} f z_{t} \in A J_{t} f z_{t}=A z_{t},
$$


which implies that

$$
\frac{1}{t}\left\langle f z_{t}-J_{t} f z_{t}, J\left(z_{t}-u\right)\right\rangle=\left\langle A_{t} f z_{t}, J\left(z_{t}-u\right)\right\rangle \geq 0 .
$$

Thus, we have

$$
\left\langle z_{t}-f z_{t}, J\left(z_{t}-u\right)\right\rangle \leq 0 .
$$

It is easy to see from Lemma 2.11 (b) that there exists a subsequence $\left\{z_{n_{i}}\right\}$ of $\left\{z_{n}\right\}$ such that $\left\{z_{n_{i}}\right\}$ converges strongly to $u$.

In order to prove that $\left\{z_{n}\right\}$ converges strongly to an element of $A^{-1} 0$, assume that there is another subsequence $\left\{z_{n_{j}}\right\}$ of $\left\{z_{n}\right\}$ such that $z_{n_{j}} \rightarrow \tilde{u}$. Since $z_{n}-J_{r} z_{n} \rightarrow 0$, it follows from the continuity of $T$ and the fact $z_{n_{i}} \rightarrow z$ that $\tilde{u} \in F\left(J_{r}\right)$. Using (3.6), we have that

$$
\left\langle z_{t}-f z_{t}, J\left(z_{t}-v\right)\right\rangle \leq 0 \text { for all } v \in F\left(J_{r}\right) .
$$

By norm to weak* uniform continuity of $J$, we obtain

$$
\langle u-f u, J(u-\tilde{u})\rangle \leq 0
$$

and

$$
\langle\tilde{u}-f \tilde{u}, J(\tilde{u}-u)\rangle \leq 0 .
$$

Adding these two inequalities yields that

$$
\langle u-\tilde{u}+f \tilde{u}-f u, J(u-\tilde{u})\rangle \leq 0,
$$

from which it follows that

$$
\begin{aligned}
\|u-\tilde{u}\|^{2} & \leq\|f u-f \tilde{u}\|\|u-\tilde{u}\| \\
& \leq[\|u-\tilde{u}\|-\psi(\|u-\tilde{u}\|)]\|u-\tilde{u}\| .
\end{aligned}
$$

Thus, we see that $u=\tilde{u}$ and hence $\left\{z_{n}\right\}$ converges strongly to $u$.

We finally prove that the path $\left\{z_{t}\right\}$ converges strongly. Towards this end, we assume that $\left\{t_{n^{\prime}}\right\}$ is another subsequence in $(0, \infty)$ such that $z_{t_{n^{\prime}}} \rightarrow u^{\prime}$ as $t_{n^{\prime}} \rightarrow \infty$. By (3.4), we obtain $u^{\prime} \in F\left(J_{r}\right)$. From (3.7), we have that

$$
\left\langle u-f u, J\left(u-u^{\prime}\right)\right\rangle \leq 0 \text { and }\left\langle u^{\prime}-f u^{\prime}, J\left(u^{\prime}-u\right)\right\rangle \leq 0 .
$$

We must have $u=u^{\prime}$. Therefore, $\left\{z_{t}\right\}$ converges strongly to $u \in A^{-1} 0$.

(c) Since $z_{t} \rightarrow u \in A^{-1} 0$ as $t \rightarrow \infty$, it follows from 3.7 that

$$
\langle u-f u, J(u-v)\rangle \leq 0 \text { for all } v \in A^{-1} 0 .
$$

Remark 3.2. Similar results can be found in Reich [18, Takahashi and Ueda [23] and $\mathrm{Xu}[25]$ when $f$ is constant.

Remark 3.3. If $A^{-1} 0$ is nonempty, then the path $\left\{z_{t}\right\}$ defined by 3.3 is bounded. Indeed, for $v \in A^{-1} 0$ and $t>0$, we have

$$
\begin{aligned}
\left\|z_{t}-v\right\| & \leq\left\|J_{t} f z_{t}-J_{t} v\right\| \leq\left\|f z_{t}-v\right\| \\
& \leq\left\|f z_{t}-f v\right\|+\|f v-v\| \\
& \leq\left\|z_{t}-v\right\|-\psi\left(\left\|z_{t}-v\right\|\right)+\|f v-v\|,
\end{aligned}
$$

which implies that

$$
\psi\left(\left\|z_{t}-v\right\|\right) \leq\|f v-v\| .
$$


Suppose $\left\{z_{t}-v\right\}$ is not bounded. Then there exists a sequence $\left\{t_{k}\right\}$ in $(0, \infty)$ with $t_{k} \rightarrow \infty$ as $k \rightarrow \infty$ such that

$$
\left\|z_{t_{k}}-v\right\|>k \text { for all } k \in \mathbb{N} \text {. }
$$

Since $\psi$ is nondecreasing and $\lim _{t \rightarrow \infty} \psi(t)=\infty$, it follows from (3.8) that

$$
\psi(k)<\psi\left(\left\|z_{t_{k}}-v\right\|\right) \leq\|f v-v\|,
$$

a contradiction.

Corollary 3.4. Let $X$ be a reflexive Banach space with a uniformly Gâteaux differentiable norm, $C$ a nonempty closed convex subset of $X$ and $A \subset X \times X$ an accretive operator such that $A^{-1} 0 \neq \emptyset$ and $\overline{D(A)} \subset C \subset \bigcap_{t>0} R(I+t A)$. Suppose that every closed convex bounded subset of $C$ has fixed point property for nonexpansive self-mappings. Then we have the following:

(a) $A^{-1} 0$ is a sunny nonexpansive retract of $C$,

(b) for each $x \in C,\left\{J_{t} x\right\}$ converges strongly to $Q_{A^{-1} 0} x$ as $t \rightarrow \infty$, where $Q_{A^{-1} 0}$ is the sunny nonexpansive retraction from $C$ onto $A^{-1} 0$.

Proof. (a) In this case the mapping $f: C \rightarrow C$ defined by $f z=x_{0}$ for all $z \in C$ is a weakly contraction. It is easy to see from Theorem 3.1 that $\left\{z_{t}=J_{t} x_{0}\right\}$ converges strongly to $u \in A^{-1} 0$ as $t \rightarrow \infty$.

Now, let $x \in C$. Then $\lim _{t \rightarrow \infty} J_{t} x \in A^{-1} 0$ and there exists a mapping $Q$ from $C$ onto $A^{-1} 0$ defined by $\lim _{t \rightarrow \infty} J_{t} x=Q x$ since $x$ is an arbitrary element of $C$. From (3.7), we have

$$
\left\langle z_{t}-x, J\left(z_{t}-v\right)\right\rangle \leq 0 \text { for all } v \in A^{-1} 0
$$

it follows that

$$
\langle Q x-x, J(Q x-v)\rangle \leq 0 \text { for all } v \in A^{-1} 0 .
$$

Therefore, $Q$ is the sunny nonexpansive retraction by Proposition 2.5

(b) It follows from part (a).

We now replace the fixed point property assumption, mentioned in Theorem 3.1 by imposing certain conditions on the space $X$ or on the set $C$.

Theorem 3.5. Let $X$ be a reflexive Banach space with a uniformly Gâteaux differentiable norm, $C$ a nonempty closed convex subset of $X, f: C \rightarrow C$ a weakly contraction mapping with the function $\psi$ and $A \subset X \times X$ an accretive operator with $A^{-1} 0 \neq \emptyset$ such that $\overline{D(A)} \subset C \subset \bigcap_{t>0} R(I+t A)$. Suppose that $X$ is strictly convex or $C$ has normal structure. Then we have the following:

(a) for each $t>0, J_{t} f$ has a unique fixed point $z_{t} \in C$;

(b) $\left\{z_{t}\right\}$ converges strongly to $u \in A^{-1} 0$ as $t \rightarrow \infty$;

(c) $u$ is the unique solution of the variational inequality:

$$
\text { find } \tilde{x} \in A^{-1} 0 \text { such that }\langle(I-f) \tilde{x}, J(\tilde{x}-v)\rangle \leq 0 \text { for all } v \in A^{-1} 0 \text {. }
$$

Proof. Note that Remark 3.3 implies that $\left\{z_{t}\right\}$ is bounded. Observe that

$$
\begin{aligned}
\left\|f z_{t}-v\right\| & \leq\left\|f z_{t}-f v\right\|+\|f v-v\| \\
& \leq\left\|z_{t}-v\right\|-\psi\left(\left\|z_{t}-v\right\|\right)+\|f v-v\| \\
& \leq\left\|z_{t}-v\right\|+\|f v-v\|,
\end{aligned}
$$


which implies by boundedness of $\left\{z_{t}\right\}$ that $\left\{f z_{t}\right\}$ is bounded.

To be able to use the argument of the proof of Theorem 3.1. we just need to show that the set $M$ defined by (2.1) has a fixed point of $J_{r}$. Suppose that $X$ is strictly convex. Since $A^{-1} 0 \neq \emptyset$, let $v \in F\left(J_{r}\right)$. Then the set $M_{0}$ defined by

$$
M_{0}=\left\{u \in M:\|u-v\|=\inf _{x \in M}\|x-v\|\right\}
$$

is a singleton since $X$ is strictly convex. Let $M_{0}=\left\{u_{0}\right\}$ for some $u_{0} \in M$. Observe that

$$
\left\|J_{r} u_{0}-v\right\|=\left\|J_{r} u_{0}-J_{r} v\right\| \leq\left\|u_{0}-v\right\|=\inf _{x \in M}\|x-v\| .
$$

Therefore, $J_{r} u_{0}=u_{0}$.

If $C$ has normal structure, then the Kirk's fixed point theorem implies that $J_{r}$ has a fixed point in $M$. We now follow the proof of Theorem 3.1 .

Next, we derive a result for existence of solutions of $V I_{F(T)}(C, I-f)$ in a reflexive Banach space when $D$ is a set of fixed points of nonexpansive self-mapping $\mathrm{T}$ on $\mathrm{C}$ and $f: C \rightarrow C$ is a weakly contraction.

Theorem 3.6. Let $X$ be a reflexive Banach space with a uniformly Gâteaux differentiable norm, $C$ a nonempty closed convex subset of $X, f: C \rightarrow C$ a weakly contraction mapping with the function $\psi$ and $T: C \rightarrow C$ a nonexpansive mapping such that $F(T) \neq \emptyset$. Suppose that every closed convex bounded subset of $C$ has fixed point property for nonexpansive self-mappings. Then we have the following:

(a) For each $t \in(0,1)$, the mapping defined by $T_{t}^{f}: C \rightarrow C$ by

$$
T_{t}^{f} x=t f x+(1-t) T x, \quad x \in C
$$

has a unique fixed point $z_{t} \in C$ such that $z_{t} \rightarrow u \in F(T)$ as $t \rightarrow 0^{+}$,

(b) $u$ is the unique solution of the variational inequality:

find $\tilde{x} \in F(T)$ such that $\langle(I-f) \tilde{x}, J(\tilde{x}-v)\rangle \leq 0$ for all $v \in F(T)$.

Proof. (a) It is obvious that there exists a unique fixed point $z_{t}$ of $T_{t}^{f}$ such that

$$
z_{t}=t f z_{t}+(1-t) T z_{t} .
$$

First, we show that $\left\{z_{t}\right\}$ is bounded. Let $v \in F(T)$. Observe that

$$
\begin{aligned}
\left\|z_{t}-v\right\| & \leq t\left\|f z_{t}-v\right\|+(1-t)\left\|T z_{t}-v\right\| \\
& \leq t\left(\left\|f z_{t}-f v\right\|+\|f v-v\|\right)+(1-t)\left\|z_{t}-v\right\| \\
& \leq t\left(\left\|z_{t}-v\right\|-\psi\left(\left\|z_{t}-v\right\|\right)+\|f v-v\|\right)+(1-t)\left\|z_{t}-v\right\|,
\end{aligned}
$$

which implies that

$$
\psi\left(\left\|z_{t}-v\right\|\right) \leq\|f v-v\|
$$

Hence $\left\{z_{t}\right\}$ satisfies 3.8 . Using the argument of Remark 3.3 , we obtain that $\left\{z_{t}\right\}$ is bounded.

It is well known from Theorem 4.6.4 of Takahashi 22 that if $T$ is nonexpansive, then $A=I-T$ is accretive and $\overline{D(A)}=C \subset \bigcap_{\lambda>0} R(I+\lambda A)$. Hence $A$ satisfies (3.1). Also from 3.9 we obtain that $z_{t}+\frac{1-t}{t}\left(z_{t}-T z_{t}\right)=f z_{t}$, which implies that $J_{\mu(t)} f z_{t}=z_{t}$, where $\mu(t)=\frac{1-t}{t}$. Therefore, $z_{t} \rightarrow z \in A^{-1} 0=F(T)$ by Theorem 3.5 .

(b) It follows from Theorem 3.5 . 
Theorem 3.7. Let $X$ be a reflexive Banach space with a uniformly Gâteaux differentiable norm, $C$ a nonempty closed convex subset of $X$ and $T: C \rightarrow C$ a nonexpansive mapping such that $F(T) \neq \emptyset$. Suppose that every closed convex bounded subset of $C$ has fixed point property for nonexpansive self-mappings. Then $F(T)$ is the sunny nonexpansive retract of $C$.

Corollary 3.8. Let $X$ be a reflexive Banach space with a uniformly Gâteaux differentiable norm, $C$ a nonempty closed convex subset of $X, f: C \rightarrow C$ a weakly contraction mapping with the function $\psi$ and $T: C \rightarrow C$ a nonexpansive mapping such that $F(T) \neq \emptyset$. For each $t \in(0,1)$, define $T_{t}^{f}: C \rightarrow C$ by

$$
T_{t}^{f} x=t f x+(1-t) T x, \quad x \in C .
$$

Suppose that $X$ is strictly convex or $C$ has normal structure. Then we have the following:

(a) $T_{t}^{f}$ has a unique fixed point $z_{t} \in C$,

(b) $\left\{z_{t}\right\}$ converges strongly to $u \in F(T)$ as $t \rightarrow 0^{+}$,

(c) $u$ is the unique solution of the variational inequality:

find $\tilde{x} \in F(T)$ such that $\langle(I-f) \tilde{x}, J(\tilde{x}-v)\rangle \leq 0$ for all $v \in F(T)$.

Remark 3.9. Corollary 3.8 improves a number of results concerning viscosity approximation methods, particularly the results of Moudafi [16] and Xu 24] in the following way:

(1) in variational inequality $V I_{F(T)}(C, I-f)$, the mapping $f$ is not necessarily a contraction,

(2) the underlying space $X$ is not necessarily Hilbert or uniformly smooth.

\section{VARIATIONAL INEQUALITIES INVOLVING NONEXPANSIVE MAPPINGS}

In this section we give sufficient conditions on $X, C$ and $T$ which provides affirmative answer of Question 1.3 .

Theorem 4.1. Let $X$ be a reflexive strictly convex smooth Banach space. Let $C$ be a nonempty closed convex bounded subset of $X$ with normal structure and let $f, T: C \rightarrow C$ be two nonexpansive mappings. If $T$ is demicompact at zero, then we have the following:

(a) there exists a sequence $\left\{z_{n}\right\}$ in $C$ such that

$$
z_{n}=t_{n} f z_{n}+\left(1-t_{n}\right) T z_{n} \text { for all } n \in \mathbb{N},
$$

where $\left\{t_{n}\right\}$ is a sequence in $(0,1)$ with $t_{n} \rightarrow 0$,

(b) the sequence $\left\{z_{n}\right\}$ in $C$ generated by 4.1) converges strongly to an element of $F(T) \cap \Omega_{F(T)}(I-f)$.

Proof. (a) For each $t \in(0,1)$, the mapping $T_{t}^{f}: C \rightarrow C$ defined by

$$
T_{t}^{f} x=t f x+(1-t) T x, x \in C
$$

is nonexpansive. Indeed, for $x, y \in C$, we have

$$
\left\|T_{t}^{f} x-T_{t}^{f} y\right\| \leq t\|f x-f y\|+(1-t)\|T x-T y\| \leq\|x-y\| .
$$


By Kirk's theorem, $F\left(T_{t}^{f}\right) \neq \emptyset$ and hence Lemma 2.8 implies that there exists the unique minimum norm solution $z_{t}$ of equation $x=T_{t}^{f} x$, i.e.,

$$
z_{t}=t f z_{t}+(1-t) T z_{t} .
$$

Observe that

$$
\left\|z_{t}-T z_{t}\right\| \leq\left\|f z_{t}-T z_{t}\right\| \leq t \operatorname{diam}(C) \rightarrow 0 \text { as } t \rightarrow 0^{+},
$$

where $\operatorname{diam}(C)$ is the diameter of $C$. Since $T$ is demicompact at zero, we may assume that $z_{t_{n}} \rightarrow z \in C$ as $n \rightarrow \infty$, where $\left\{t_{n}\right\}$ is a sequence in $(0,1)$ such that $\lim t_{n}=0$. Set $z_{n}:=z_{t_{n}}$. Then from $\sqrt{4.2}$, there exists a sequence in $C$ satisfying 4.1 .

(b) Since $z_{n}-T z_{n} \rightarrow 0$ and $z_{n} \rightarrow z$, it follows that $z \in F(T)$. So, it remains to show that $z$ is a solution of the variational inequality:

find $x \in F(T)$ such that $\langle(I-f) x, J(x-v)\rangle \leq 0$ for all $v \in F(T)$.

Let $v \in F(T)$. Observe that

$$
\begin{aligned}
\left\langle z_{t}-T z_{t}, J\left(z_{t}-v\right)\right\rangle & =\left\langle z_{t}-v+T v-T z_{t}, j\left(z_{t}-v\right)\right\rangle \\
& =\left\|z_{t}-v\right\|^{2}-\left\langle T z_{t}-T z, J\left(z_{t}-v\right)\right\rangle \\
& \geq\left\|z_{t}-v\right\|^{2}-\left\|T z_{t}-T v\right\|\left\|z_{t}-v\right\| \\
& \geq 0 .
\end{aligned}
$$

Hence from (4.2), we have

$$
\begin{aligned}
\left\langle z_{t}-f z_{t}, J\left(z_{t}-v\right)\right\rangle & =(1-t)\left\langle T z_{t}-f z_{t}, J\left(z_{t}-v\right)\right\rangle \\
& \leq(1-t)\left\langle T z_{t}-z_{t}+z_{t}-f z_{t}, J\left(z_{t}-v\right)\right\rangle,
\end{aligned}
$$

which implies form 4.3 that

$$
\left\langle z_{t}-f z_{t}, J\left(z_{t}-v\right)\right\rangle \leq 0 .
$$

and hence, we get

$$
\left\langle z_{n}-f z_{n}, J\left(z_{n}-v\right)\right\rangle \leq 0 \text { for all } n \in \mathbb{N} .
$$

Since $J$ is norm-to-weak ${ }^{*}$ continuous, it follows from $z_{n} \rightarrow z$ that $J\left(z_{n}-v\right) \rightarrow^{*}$ $J(z-v)$. Observe that

$$
\begin{aligned}
\mid\left\langle z_{n}\right. & \left.-f z_{n}, J\left(z_{n}-v\right)\right\rangle-\langle z-f z, J(z-v)\rangle \mid \\
& =\left|\left\langle z_{n}-f z_{n}-(z-f z), J\left(z_{n}-v\right)\right\rangle+\left\langle z-f z, J\left(z_{n}-v\right)-J(z-v)\right\rangle\right| \\
& \leq\left\|z_{n}-f z_{n}-(z-f z)\right\|\left\|z_{n}-v\right\|+\left|\left\langle z-A z, J\left(z_{n}-v\right)-J(z-v)\right\rangle\right| \rightarrow 0,
\end{aligned}
$$

it follows from 4.4 that

$$
\langle z-f z, J(z-v)\rangle=\lim _{n \rightarrow \infty}\left\langle z_{n}-f z_{n}, J\left(z_{n}-v\right)\right\rangle \leq 0 .
$$

It is well known that a closed convex subset of a uniformly convex Banach space has normal structure and a compact convex subset of a Banach space has normal structure.

Theorem 4.2. Let $X$ be a smooth uniformly convex Banach space. Let $C$ be a nonempty closed convex bounded subset of $X$ and let $f, T: C \rightarrow C$ be two nonexpansive mappings. If $T$ is demicompact at zero, then $F(T) \cap \Omega_{F(T)}(I-f) \neq \emptyset$. 
Corollary 4.3. Let $X$ be a reflexive strictly convex smooth Banach space. Let $C$ be a nonempty compact convex subset of $X$ and let $f, T: C \rightarrow C$ be two nonexpansive mappings. Then $F(T) \cap \Omega_{F(T)}(I-f) \neq \emptyset$.

Next, we consider the variational inequality $V I_{F(T)}(C, I-f)$ for pseudocontractive mappings $f: C \rightarrow C$.

Theorem 4.4. Let $X$ be a reflexive strictly convex smooth Banach space. Let $C$ be a nonempty closed convex bounded subset of $X$ with normal structure, $f: C \rightarrow C$ a continuous pseudocontractive mapping and $T: C \rightarrow C$ a nonexpansive mapping. If $T$ is demicompact at zero, then there exists a sequence $\left\{z_{n}\right\}$ in $C$ such that

$$
z_{n}=t_{n} f z_{n}+\left(1-t_{n}\right) T z_{n} \text { for all } n \in \mathbb{N},
$$

where $\left\{t_{n}\right\}$ is a sequence in $(0,1)$ with $t_{n} \rightarrow 0$, and the sequence $\left\{z_{n}\right\}$ in $C$ generated by (4.5) converges strongly to an element of $F(T) \cap \Omega_{F(T)}(I-f)$.

Proof. For each $t \in(0,1)$, the mapping $T_{t}^{f}: C \rightarrow C$ defined by

$$
T_{t}^{f} x=t f x+(1-t) T x, x \in C
$$

is pseudocontractive. Indeed, for $x, y \in C$, we have

$$
\begin{aligned}
\left\langle T_{t}^{f} x-T_{t}^{f} y, J(x-y)\right\rangle & =t\langle f x-f y, J(x-y)\rangle+(1-t)\langle T x-T y, J(x-y)\rangle \\
& \leq\|x-y\|^{2} .
\end{aligned}
$$

It follows from Theorem 6 of Martin 14 that $g_{t}^{f}=\left(2 I-T_{t}^{f}\right)^{-1}$ is a nonexpansive mapping from $C$ into itself with $F\left(g_{t}^{f}\right)=F\left(T_{t}^{f}\right)$. By Kirk's theorem, $F\left(g_{t}^{f}\right) \neq \emptyset$ and hence Lemma 2.8 implies that there exists the unique minimum norm solution $z_{t}$ of $x=g_{t}^{f} x$, i.e.,

$$
z_{t}=t f z_{t}+(1-t) T z_{t} .
$$

Similar to the argument of proof of Theorem 4.1. we have a sequence $\left\{z_{n}\right\}$ in $C$ such that

$$
z_{n}=t_{n} f z_{n}+\left(1-t_{n}\right) T z_{n} \text { for all } n \in \mathbb{N},
$$

where $\left\{t_{n}\right\}$ is a sequence in $(0,1)$ with $t_{n} \rightarrow 0$, and the sequence $\left\{z_{n}\right\}$ in $C$ converges strongly to an element of $F(T) \cap \Omega_{F(T)}(I-f)$.

It is well known that if $C$ is a closed convex subset of a Hilbert space $H$, then a sunny nonexpansive retraction is coincident with the metric projection mapping $P_{C}$ from $H$ onto $C$. This is not true in general, since outside Hilbert space, the metric projection mappings, although sunny, are no longer nonexpansive. From Theorem 3.8 we can derive the following result for existence of sunny nonexpansive retractions (from $C$ onto $F(T)$ ) in Banach spaces.

Theorem 4.5. Let $X$ be a reflexive Banach space whose norm is uniformly Gâteaux differentiable, $C$ a nonempty closed convex subset of $X$ and $T: C \rightarrow C$ a nonexpansive mapping with $F(T) \neq \emptyset$. Suppose that $X$ is strictly convex or $C$ has normal structure. Then $F(T)$ is a sunny nonexpansive retract of $C$.

We now turn our attention to deal with the problem of existence of solutions of $V I_{D}(C, I-f)$ by sunny nonexpansive retractions. 
Let $C$ be a convex subset of a smooth Banach space $X$ and $D$ a nonempty subset of $C$. Let $Q_{D}$ be the sunny nonexpansive retraction from $C$ onto $D$. Let $x \in C$ and $x_{0} \in D$. Then from Lemma 2.5, we have

$$
x_{0}=Q_{D} x \text { if and only if }\left\langle x_{0}-x, J\left(x_{0}-v\right)\right\rangle \leq 0 \text { for all } v \in D .
$$

Following relation $(4.6)$, we can show that the variational inequality $V I_{D}(C, I-$ $f$ ) is equivalent to the fixed point problem.

Proposition 4.6. Let $C$ be a convex subset of a smooth Banach space $X$ and $D$ a nonempty subset of $C$. Let $f: C \rightarrow C$ be a mapping and let $Q_{D}$ be the sunny nonexpansive retraction from $C$ onto $D$. Then we have the following:

(a) $\Omega_{D}(I-f)=F\left(Q_{D} f\right)$.

(b) If $f: C \rightarrow C$ is a Lipschitzian mapping with Lipschitz constant $L$, then $Q_{D} f$ is also a Lipschitzian mapping with the same Lipschitz constant.

Proof. (a) For any $z \in C$, we obtain from 4.6 that

$$
z=Q_{D} f z \text { if and only if }\langle z-f z, J(z-v)\rangle \leq 0 \text { for all } v \in D .
$$

(b) For $x, y \in C$, we have

$$
\left\|Q_{D} f x-Q_{D} f y\right\| \leq\|f x-f y\| \leq L\|x-y\| .
$$

The following result shows that the Kirk's fixed point theorem plays an important role in existence of common element of $D$ and set of solutions of variational inequality $V I_{D}(C, I-f)$ when $f: C \rightarrow C$ is a nonexpansive mapping.

Theorem 4.7. Let $X$ be a reflexive smooth Banach space, $C$ a nonempty closed convex bounded subset of $X$ with normal structure and $D$ a nonempty subset of $C$ which is a sunny nonexpansive retract of $C$. Let $f: C \rightarrow C$ be a nonexpansive mapping and let $Q_{D}$ be the sunny nonexpansive retraction from $C$ onto $D$. Then variational inequality $V I(C, I-f)$ has a solution in $D$.

Proof. Since $Q_{D} f$ is a nonexpansive mapping from $C$ into $D \subseteq C$ with $\Omega_{D}(I-$ $f)=F\left(Q_{D} f\right)$. It follows from Kirk's fixed point theorem that $F\left(Q_{D} f\right) \neq \emptyset$. Let $z \in F\left(Q_{D} f\right)$. Then $z=Q_{D} f z \in D$.

Invoking Theorem 4.5, we obtain the following existence theorem:

Theorem 4.8. Let $X$ be a reflexive Banach space whose norm is uniformly Gâteaux differentiable and $C$ a nonempty closed convex bounded subset of $X$ with normal structure. Let $f, T: C \rightarrow C$ be two nonexpansive mappings. Then variational inequality $V I(C, I-f)$ has a solution in $F(T)$.

Proof. Note that $F(T) \neq \emptyset$ by Theorem 2.4. By Theorem 4.5, there exists a sunny nonexpansive retraction $C$ onto $F(T)$. Let $Q_{F(T)}$ be the sunny nonexpansive retraction $C$ onto $F(T)$. Then the result follows from Theorem 4.7 .

Theorem 4.9. Let $X$ be a reflexive Banach space whose norm is uniformly Gâteaux differentiable and $C$ a nonempty closed convex bounded subset of $X$ with normal structure. Let $f: C \rightarrow C$ be a nonexpansive mapping and $T: C \rightarrow C$ a continuous pseudocontractive mapping. Then variational inequality $V I_{F(T)}(C, I-f)$ has a solution in $F(T)$. 
Proof. Since $g=(2 I-T)^{-1}$ is nonexpansive mapping from $C$ into itself with $F(g)=$ $F(T)$. Then $F(g) \neq \emptyset$ by Theorem 2.4 . Let $Q_{F(g)}$ be the sunny nonexpansive retraction from $C$ onto $F(g)$. Then the result follows from Theorem 4.7 .

\section{Strong Convergence of iterative Algorithms}

In this section, we apply the results investigated in Sections 3 and 4 for computing solutions of variational inequalities $V I_{C}(I-f)$ in Banach spaces when $f$ is a weakly contraction or nonexpansive mapping. In order to prove our main convergence theorems, we start with a key preliminary result.

Lemma 5.1. Let $C$ be a nonempty closed convex subset of a smooth Banach space $X, f: C \rightarrow C$ a weakly contraction mapping with the function $\psi$ and $\left\{T_{n}\right\}$ a sequence of nonexpansive self-mappings on $C$ such that $\bigcap_{n \in \mathbb{N}} F\left(T_{n}\right) \neq \emptyset$. For given $x_{1} \in C$, let $\left\{x_{n}\right\}$ be a sequence in $C$ generated by algorithm:

$$
x_{n+1}=\alpha_{n} f x_{n}+\left(1-\alpha_{n}\right) T_{n} x_{n} \text { for all } \in \mathbb{N},
$$

where $\left\{\alpha_{n}\right\}$ is a sequence in $[0,1]$ such that $\sum_{n=1}^{\infty} \alpha_{n}=\infty$. Then we have the following:

(a) If $\inf \left\{\psi\left(\left\|x_{n}-p\right\|\right) /\left\|x_{n}-p\right\|: x_{n} \neq p, n \in \mathbb{N}\right\}=\delta>0$ for $p \in \bigcap_{n \in \mathbb{N}} F\left(T_{n}\right)$, then $\left\{x_{n}\right\}$ is bounded.

(b) If $\left\{x_{n}\right\}$ is bounded and there exists a point $z \in \bigcap_{n \in \mathbb{N}} F\left(T_{n}\right)$ such that

$$
\limsup _{n \rightarrow \infty}\left\langle f z-z, J\left(x_{n}-z\right)\right\rangle \leq 0,
$$

then $\left\{x_{n}\right\}$ converges strongly to $z$.

Proof. (a) Let $p \in \bigcap_{n \in \mathbb{N}} F\left(T_{n}\right)$. From (5.1), we have

$$
\begin{aligned}
\left\|x_{n+1}-p\right\| & =\left\|\alpha_{n}\left(f x_{n}-f p+f p-p\right)+\left(1-\alpha_{n}\right)\left(T_{n} x_{n}-p\right)\right\| \\
& \leq \alpha_{n}\left\|f x_{n}-f p\right\|+\alpha_{n}\|f p-p\|+\left(1-\alpha_{n}\right)\left\|T_{n} x_{n}-p\right\| \\
& \leq \alpha_{n}\left(\left\|x_{n}-p\right\|-\psi\left(\left\|x_{n}-p\right\|\right)\right)+\alpha_{n}\|f p-p\|+\left(1-\alpha_{n}\right)\left\|x_{n}-p\right\| \\
& =\left\|x_{n}-p\right\|-\alpha_{n} \psi\left(\left\|x_{n}-p\right\|\right)+\alpha_{n}\|f p-p\| .
\end{aligned}
$$

Since $0<\delta=\inf \left\{\psi\left(\left\|x_{n}-p\right\|\right) /\left\|x_{n}-p\right\|: x_{n} \neq p, n \in \mathbb{N}\right\}$, it follows from (5.3) that

$$
\begin{aligned}
\left\|x_{n+1}-p\right\| & \leq\left(1-\alpha_{n} \delta\right)\left\|x_{n}-p\right\|+\alpha_{n}\|f p-p\| \\
& \leq \max \left\{\left\|x_{n}-p\right\|,\|f p-p\| / \delta\right\} \\
& \vdots \\
& \leq \max \left\{\left\|x_{1}-p\right\|,\|f p-p\| / \delta\right\} .
\end{aligned}
$$

Consequently, $\left\{x_{n}\right\}$ is bounded.

(b) From $(5.2)$, there exists a sequence $\left\{\gamma_{n}\right\}$ in $(0, \infty)$ with $\lim _{n \rightarrow \infty} \gamma_{n}=0$ such that

$$
\left\langle f z-z, J\left(x_{n+1}-z\right)\right\rangle \leq \gamma_{n} \text { for all } \in \mathbb{N} \text {. }
$$


From (5.1), we obtain

$$
\begin{aligned}
\left\|x_{n+1}-z\right\|^{2}= & \left\langle\alpha_{n}\left(f x_{n}-f z+f z-z\right)+\left(1-\alpha_{n}\right)\left(T_{n} x_{n}-z\right), J\left(x_{n+1}-z\right)\right\rangle \\
\leq & \left\|\left(1-\alpha_{n}\right)\left(T_{n} x_{n}-z\right)+\alpha_{n}\left(f x_{n}-f z\right)\right\|\left\|x_{n+1}-z\right\| \\
& +\alpha_{n}\left\langle f z-z, J\left(x_{n+1}-z\right)\right\rangle \\
\leq & \frac{1}{2}\left[\left\|\left(1-\alpha_{n}\right)\left(T_{n} x_{n}-z\right)+\alpha_{n}\left(f x_{n}-f z\right)\right\|^{2}+\left\|x_{n+1}-z\right\|^{2}\right]+\alpha_{n} \gamma_{n} \\
\leq & \frac{1}{2}\left[\left(1-\alpha_{n}\right)\left\|x_{n}-z\right\|+\alpha_{n}\left(\left\|x_{n}-z\right\|-\psi\left(\left\|x_{n}-z\right\|\right)\right)\right]^{2} \\
& +\frac{1}{2}\left\|x_{n+1}-z\right\|^{2}+\alpha_{n} \gamma_{n},
\end{aligned}
$$

which implies that

$$
\begin{aligned}
\left\|x_{n+1}-z\right\|^{2} \leq & \left\|x_{n}-z\right\|^{2}-2 \alpha_{n} \psi\left(\left\|x_{n}-z\right\|\right)\left\|x_{n}-z\right\|+\alpha_{n}^{2}\left(\psi\left(\left\|x_{n}-z\right\|\right)\right)^{2} \\
& +2 \alpha_{n} \gamma_{n} \\
\leq & \left\|x_{n}-z\right\|^{2}-2 \alpha_{n} \psi\left(\left\|x_{n}-z\right\|\right)\left\|x_{n}-z\right\|+\alpha_{n}^{2}\left(\psi\left(K_{3}\right)\right)^{2}+2 \alpha_{n} \gamma_{n}
\end{aligned}
$$

for some $K_{3}>0$ since $\left\{\left\|x_{n}-z\right\|\right\}$ is bounded. Thus, for $\lambda_{n}=\left\|x_{n}-z\right\|^{2}$, we obtain the following recursive inequality:

$$
\lambda_{n+1} \leq \lambda_{n}-\alpha_{n} \phi\left(\lambda_{n}\right)+\beta_{n},
$$

where $\beta_{n}=\alpha_{n}\left(K_{3} \alpha_{n}+2 \gamma_{n}\right)$ and $\phi(t)=2 \sqrt{t} \psi(\sqrt{t})$. Therefore, $\left\{x_{n}\right\}$ converges strongly to $z$ by Lemma 2.9 .

Remark 5.2. If $f: C \rightarrow C$ is a contraction mapping with Lipschitz constant $k \in(0,1)$, then the assumption $\inf \left\{\psi\left(\left\|x_{n}-p\right\|\right) /\left\|x_{n}-p\right\|: x_{n} \neq p, n \in \mathbb{N}\right\}=\delta>0$ for $p \in \bigcap_{n \in \mathbb{N}} F\left(T_{n}\right)$ is satisfied with $\delta=k$. Thus, the sequence $\left\{x_{n}\right\}$ defined by (5.1) is always bounded for contraction mappings $f: C \rightarrow C$.

Theorem 5.3. Let $X$ be a reflexive Banach space with a uniformly Gâteaux differentiable norm, $C$ a nonempty closed convex subset of $X, f: C \rightarrow C$ a weakly contraction mapping with the function $\psi$ and $A \subset X \times X$ an accretive operator with resolvent $J_{t}$ for $t>0$ such that $A^{-1} 0 \neq \emptyset$ and $\overline{D(A)} \subset C \subset \bigcap_{t>0} R(I+t A)$. Suppose that $X$ is strictly convex or $C$ has normal structure. For given $x_{1} \in C$, let $\left\{x_{n}\right\}$ be a sequence in $C$ generated by algorithm:

$$
x_{n+1}=\alpha_{n} f x_{n}+\left(1-\alpha_{n}\right) J_{t_{n}} x_{n} \text { for all } n \in \mathbb{N},
$$

where $\left\{\alpha_{n}\right\}$ is a sequence in [0,1] and $\left\{t_{n}\right\}$ is a sequence in $(0, \infty)$ such that

$$
\lim _{n \rightarrow \infty} \alpha_{n}=0, \sum_{n=1}^{\infty} \alpha_{n}=\infty \text { and } \lim _{n \rightarrow \infty} t_{n}=\infty .
$$

Then $\left\{x_{n}\right\}$ converges strongly to the unique solution of the variational inequality:

find $\tilde{x} \in A^{-1} 0$ such that $\langle(I-f) \tilde{x}, J(\tilde{x}-v)\rangle \leq 0$ for all $v \in A^{-1} 0$ provided that $\inf \left\{\psi\left(\left\|x_{n}-p\right\|\right) /\left\|x_{n}-p\right\|: x_{n} \neq p, n \in \mathbb{N}\right\}=\delta>0$ for $p \in A^{-1} 0$.

Proof. Note that $\left\{x_{n}\right\}$ is bounded by Lemma 5.1(a) and hence both $\left\{f x_{n}\right\}$ and $\left\{J_{t_{n}} x_{n}\right\}$ are bounded. Hence form 5.5, we have

$$
\left\|x_{n+1}-J_{t_{n}} x_{n}\right\|=\alpha_{n}\left\|f x_{n}-J_{t_{n}} x_{n}\right\| \rightarrow 0 \text { as } n \rightarrow \infty .
$$


Observe that

$$
\left\|A_{t_{n}} x_{n}\right\|=\frac{1}{t_{n}}\left\|x_{n}-J_{t_{n}} x_{n}\right\| \rightarrow 0 \text { as } n \rightarrow \infty .
$$

By Theorem 3.5 for each $t>0$, the mapping $T_{t}^{f}: C \rightarrow C$ defined by

$$
T_{t}^{f} x=J_{t} f x, x \in C
$$

has a unique fixed point $z_{t} \in C$ such that $\left\{z_{t}\right\}$ converges strongly to an element $z \in A^{-1} 0$ as $t \rightarrow \infty$, and $z$ is the unique solution of the variational inequality $V I_{A^{-1} 0}(C, I-f)$. From 3.5 we have

$$
\frac{1}{t}\left(f z_{t}-z_{t}\right)=A_{t} f z_{t} \in A J_{t} f z_{t}=A z_{t}
$$

it follows from the fact $A_{t_{n}} x_{n} \in A J_{t_{n}} x_{n}$ that

$$
0 \leq\left\langle A_{t_{n}} x_{n}-\frac{1}{t}\left(f z_{t}-z_{t}\right), J\left(J_{t_{n}} x_{n}-z_{t}\right)\right\rangle .
$$

By boundedness of $\left\{J_{t_{n}} x_{n}-z_{t}\right\}$, we have

$$
\begin{aligned}
\left\langle f z_{t}-z_{t}, J\left(J_{t_{n}} x_{n}-z_{t}\right)\right\rangle & \leq t\left\langle A_{t_{n}} x_{n}, J\left(J_{t_{n}} x_{n}-z_{t}\right)\right\rangle \\
& \leq t\left\|A_{t_{n}} x_{n}\right\|\left\|J_{t_{n}} x_{n}-z_{t}\right\| \\
& \leq t\left\|A_{t_{n}} x_{n}\right\| K_{4}
\end{aligned}
$$

for some $K_{4}>0$. Since $\left\|A_{t_{n}} x_{n}\right\| \rightarrow 0$ as $n \rightarrow \infty$, we see that

$$
\limsup _{n \rightarrow \infty}\left\langle f z_{t}-z_{t}, J\left(J_{t_{n}} x_{n}-z_{t}\right)\right\rangle \leq 0 .
$$

Further, since $z_{t} \rightarrow z$ as $t \rightarrow \infty$, the set $\left\{z_{t}-x_{n}\right\}$ is bounded and the duality mapping $J$ is norm-to-weak* uniformly continuous on bounded subsets of $X$, it follows that

$$
\begin{aligned}
\mid\langle f z- & \left.z, J\left(J_{t_{n}} x_{n}-z\right)\right\rangle-\left\langle f z_{t}-z_{t}, J\left(J_{t_{n}} x_{n}-z_{t}\right)\right\rangle \mid \\
= & \mid\left\langle f z-z, J\left(J_{t_{n}} x_{n}-z\right)-J\left(J_{t_{n}} x_{n}-z_{t}\right)\right\rangle \\
& +\left\langle f z-z-\left(f z_{t}-z_{t}\right), J\left(J_{t_{n}} x_{n}-z_{t}\right)\right\rangle \mid \\
\leq & \left|\left\langle f z-z, J\left(J_{t_{n}} x_{n}-z\right)-J\left(J_{t_{n}} x_{n}-z_{t}\right)\right\rangle\right| \\
& +\left\|f z-z-\left(f z_{t}-z_{t}\right)\right\|\left\|x_{n}-z_{t}\right\| \rightarrow 0 \text { as } t \rightarrow \infty .
\end{aligned}
$$

Let $\varepsilon>0$. Then there exists $t_{0}>0$ such that

$\left\langle f z-z, J\left(J_{t_{n}} x_{n}-z\right)\right\rangle\left\langle\left\langle f z_{t}-z_{t}, J\left(J_{t_{n}} x_{n}-z_{t}\right)\right\rangle+\varepsilon\right.$ for all $n \in \mathbb{N}$ and $t \geq t_{0}$.

Using (5.6), we get

$$
\begin{aligned}
\limsup _{n \rightarrow \infty}\left\langle f z-z, J\left(J_{t_{n}} x_{n}-z\right)\right\rangle & \leq \limsup _{n \rightarrow \infty}\left\langle f z_{t}-z_{t}, J\left(J_{t_{n}} x_{n}-z_{t}\right)\right\rangle+\varepsilon \\
& \leq \varepsilon .
\end{aligned}
$$

Since $\varepsilon$ is arbitrary, we obtain that

$$
\limsup _{n \rightarrow \infty}\left\langle f z-z, J\left(J_{t_{n}} x_{n}-z\right)\right\rangle \leq 0 .
$$

Since $x_{n+1}-J_{t_{n}} x_{n} \rightarrow 0$ as $n \rightarrow \infty$ and the duality mapping $J$ is norm-to-weak* uniformly continuous on bounded subsets of $X$, it follows that

$$
\lim _{n \rightarrow \infty}\left|\left\langle f z-z, J\left(x_{n+1}-z\right)\right\rangle-\left\langle f z-z, J\left(J_{t_{n}} x_{n}-z\right)\right\rangle\right|=0 .
$$


Therefore,

$$
\limsup _{n \rightarrow \infty}\left\langle f z-z, J\left(x_{n+1}-z\right)\right\rangle \leq 0 .
$$

So, there exists a sequence $\left\{\gamma_{n}\right\}$ in $(0, \infty)$ satisfying (5.4) such that $\lim _{n \rightarrow \infty} \gamma_{n}=0$. Therefore, since $z \in F\left(J_{t_{n}}\right)$ for all $n \in \mathbb{N},\left\{x_{n}\right\}$ converges strongly to $z$ by Lemma 5.1 .

We now give a more general proximal point algorithm for resolvent operators in a Banach space which provides an affirmative answer of Question 1.5 .

Theorem 5.4. Let $X$ be a reflexive Banach space with a uniformly Gâteaux differentiable norm, $C$ a nonempty closed convex subset of $X, f: C \rightarrow C$ a weakly contraction mapping with the function $\psi$ and $A \subset X \times X$ an accretive operator with resolvent $J_{t}$ for $t>0$ such that $A^{-1} 0 \neq \emptyset$ and $\overline{D(A)} \subset C \subset \bigcap_{t>0} R(I+t A)$. Suppose that $X$ is strictly convex or $C$ has normal structure. For given $x_{1} \in C$, let $\left\{x_{n}\right\}$ be a sequence in $C$ generated by algorithm:

$$
x_{n+1}=\alpha_{n} f x_{n}+\left(1-\alpha_{n}\right) J_{t_{n}} x_{n} \text { for all } n \in \mathbb{N},
$$

where $\left\{\alpha_{n}\right\}$ is a sequence in $[0,1]$ and $\left\{t_{n}\right\}$ is a sequence in $(0, \infty)$ such that

$$
\lim _{n \rightarrow \infty} \alpha_{n}=0, \sum_{n=1}^{\infty} \alpha_{n}=\infty \text { and } \lim _{n \rightarrow \infty} t_{n}=\infty .
$$

If $\left\{x_{n}\right\}$ is bounded, then it converges strongly to the unique solution of the variational inequality:

$$
\text { find } \tilde{x} \in A^{-1} 0 \text { such that }\langle(I-f) \tilde{x}, J(\tilde{x}-v)\rangle \leq 0 \text { for all } v \in A^{-1} 0 \text {. }
$$

The following result improves and unifies several known results that were obtained using stronger assumptions (see e.g. Theorem 2.5 of Benavides, Acedo and $\mathrm{Xu}$ [2, Theorem 2 of Kim and Xu [9], Theorem 3.1 of Mainge [13, and Theorem 4.2 of Nakajo [17]).

Corollary 5.5. Let $X$ be a reflexive Banach space with uniformly Gâteaux differentiable norm, $C$ a nonempty closed convex subset of $X, f: C \rightarrow C$ a contraction mapping with Lipschitz constant $k \in[0,1)$ and $A \subset X \times X$ an accretive operator with resolvent $J_{t}$ for $t>0$ such that $A^{-1} 0 \neq \emptyset$ and $\overline{D(A)} \subset C \subset \bigcap_{t>0} R(I+t A)$. Suppose that $X$ is strictly convex or $C$ has normal structure. For given $x_{1} \in C$, let $\left\{x_{n}\right\}$ be a sequence in $C$ generated by algorithm:

$$
x_{n+1}=\alpha_{n} f x_{n}+\left(1-\alpha_{n}\right) J_{t_{n}} x_{n} \text { for all } n \in \mathbb{N},
$$

where $\left\{\alpha_{n}\right\}$ is a sequence in [0,1] and $\left\{t_{n}\right\}$ is a sequence in $(0, \infty)$ such that

$$
\lim _{n \rightarrow \infty} \alpha_{n}=0, \sum_{n=1}^{\infty} \alpha_{n}=\infty \text { and } \lim _{n \rightarrow \infty} t_{n}=\infty .
$$

Then $\left\{x_{n}\right\}$ converges strongly to the unique solution of the variational inequality:

$$
\text { find } \tilde{x} \in A^{-1} 0 \text { such that }\langle(I-f) \tilde{x}, J(\tilde{x}-v)\rangle \leq 0 \text { for all } v \in A^{-1} 0 \text {. }
$$

Theorem 5.6. Let $X$ be a reflexive Banach space whose norm is uniformly Gâteaux differentiable, $C$ a nonempty closed convex subset of $X, f: C \rightarrow C$ a weakly contraction mapping with the function $\psi$ and $T: C \rightarrow C$ a nonexpansive mapping with $F(T) \neq \emptyset$. Suppose that $X$ is strictly convex or $C$ has normal structure. Let $\left\{\alpha_{n}\right\}$ be a sequence in $[0,1]$ such that 


$$
\lim _{n \rightarrow \infty} \alpha_{n}=0, \sum_{n=1}^{\infty} \alpha_{n}=\infty \text { and } \lim _{n \rightarrow \infty} \frac{\left|\alpha_{n}-\alpha_{n-1}\right|}{\alpha_{n}}=0 .
$$

For given $x_{1} \in C$, let $\left\{x_{n}\right\}$ be a sequence in $C$ generated by algorithm:

$$
x_{n+1}=\alpha_{n} f x_{n}+\left(1-\alpha_{n}\right) T x_{n} \text { for all } \in \mathbb{N} .
$$

If $\left\{x_{n}\right\}$ is bounded, then it converges strongly to the unique solution of the variational inequality:

find $\tilde{x} \in F(T)$ such that $\langle(I-f) \tilde{x}, J(\tilde{x}-v)\rangle \leq 0$ for all $v \in F(T)$.

Proof. Boundedness of $\left\{x_{n}\right\}$ implies that both $\left\{f x_{n}\right\}$ and $\left\{T x_{n}\right\}$ are bounded. Hence form (5.7), we have

$$
\left\|x_{n+1}-T x_{n}\right\|=\alpha_{n}\left\|f x_{n}-T x_{n}\right\| \rightarrow 0 \text { as } n \rightarrow \infty .
$$

Observe that

$$
\begin{aligned}
\left\|x_{n+1}-x_{n}\right\|= & \left\|\alpha_{n} f x_{n}+\left(1-\alpha_{n}\right) T x_{n}-\left(\alpha_{n-1} f x_{n-1}+\left(1-\alpha_{n-1}\right) T x_{n-1}\right)\right\| \\
\leq & \left\|\left(1-\alpha_{n}\right)\left(T x_{n}-T x_{n-1}\right)+\left(\alpha_{n}-\alpha_{n-1}\right)\left(f x_{n-1}-T x_{n-1}\right)\right\| \\
& +\alpha_{n}\left\|f x_{n}-f x_{n-1}\right\| \\
\leq & \left(1-\alpha_{n}\right)\left\|x_{n}-x_{n-1}\right\|+\alpha_{n}\left(\left\|x_{n}-x_{n-1}\right\|-\psi\left(\left\|x_{n}-x_{n-1}\right\|\right)\right) \\
& +\left|\alpha_{n}-\alpha_{n-1}\right| K_{5} \\
= & \left\|x_{n}-x_{n-1}\right\|-\alpha_{n} \psi\left(\left\|x_{n}-x_{n-1}\right\|\right)+\left|\alpha_{n}-\alpha_{n-1}\right| K_{5}
\end{aligned}
$$

for some constant $K_{5}>0$. Since $\lim _{n \rightarrow \infty} \frac{\left|\alpha_{n}-\alpha_{n-1}\right|}{\alpha_{n}}=0$, it follows from Lemma 2.9 that $\left\|x_{n+1}-x_{n}\right\| \rightarrow 0$ as $n \rightarrow \infty$. Hence

$$
\left\|x_{n}-T x_{n}\right\| \leq\left\|x_{n}-x_{n+1}\right\|+\left\|x_{n+1}-T x_{n}\right\| \rightarrow 0 \text { as } n \rightarrow \infty .
$$

For each $t \in(0,1)$, define $T_{t}^{f}: C \rightarrow C$ by

$$
T_{t}^{f} x=t f x+(1-t) T x, \quad x \in C .
$$

Note that Theorem 3.8 implies that $T_{t}^{f}$ has a unique fixed point $z_{t} \in C$ such that $\left\{z_{t}\right\}$ converges strongly to an element $z \in F(T)$ as $t \rightarrow 0^{+}$, and $z$ is the unique solution of the variational inequality $V I_{F(T)}(C, I-f)$.

Since $\left\|x_{n}-T x_{n}\right\| \rightarrow 0$ as $n \rightarrow \infty$ and $\left\{z_{t}\right\}$ converges strongly to $z \in F(T)$, it follows from Lemma 2.12 that $\limsup _{n \rightarrow \infty}\left\langle f z-z, J\left(x_{n}-z\right)\right\rangle \leq 0$. Since $z \in$ $F(T)$ and $(5.2)$ is satisfied, then we conclude from Lemma 5.1 that $\left\{x_{n}\right\}$ converges strongly to $z$.

As a direct consequence of Theorem 5.6 , we have the following:

Corollary 5.7. Let $X$ be a reflexive Banach space whose norm is uniformly Gâteaux differentiable, $C$ a nonempty closed convex subset of $X, f: C \rightarrow C$ a contraction mapping with Lipschitz constant $k \in[0,1)$, and $T: C \rightarrow C$ a nonexpansive mapping with $F(T) \neq \emptyset$. Suppose that $X$ is strictly convex or $C$ has normal structure. Let $\left\{\alpha_{n}\right\}$ be a sequence in $[0,1]$ such that

$$
\lim _{n \rightarrow \infty} \alpha_{n}=0, \sum_{n=1}^{\infty} \alpha_{n}=\infty \text { and } \lim _{n \rightarrow \infty} \frac{\left|\alpha_{n}-\alpha_{n-1}\right|}{\alpha_{n}}=0 .
$$


For given $x_{1} \in C$, let $\left\{x_{n}\right\}$ be a sequence in $C$ generated by the functional Halpern iteration process:

$$
x_{n+1}=\alpha_{n} f x_{n}+\left(1-\alpha_{n}\right) T x_{n} \text { for all } n \in \mathbb{N} .
$$

Then $\left\{x_{n}\right\}$ converges strongly to the unique solution of the variational inequality:

find $\tilde{x} \in F(T)$ such that $\langle(I-f) \tilde{x}, J(\tilde{x}-v)\rangle \leq 0$ for all $v \in F(T)$.

Remark 5.8. Corollary 5.7 improves a number of results concerning viscosity approximation methods, particularly the results of Moudafi [16] and Xu 24] in the following way:

(1) in variational inequality $V I_{F(T)}(C, I-f)$, the mapping $f$ is not necessarily contraction,

(2) the underlying space $X$ is not necessarily Hilbert or uniformly smooth.

Corollary 5.9. Let $X$ be a reflexive Banach space whose norm is uniformly Gâteaux differentiable, $C$ a nonempty closed convex subset of $X$, and $T: C \rightarrow C$ a nonexpansive mapping with $F(T) \neq \emptyset$. Suppose that $X$ is strictly convex or $C$ has normal structure. Let $\left\{\alpha_{n}\right\}$ be a sequence in [0,1] such that

$$
\lim _{n \rightarrow \infty} \alpha_{n}=0, \sum_{n=1}^{\infty} \alpha_{n}=\infty \text { and } \lim _{n \rightarrow \infty} \frac{\left|\alpha_{n}-\alpha_{n-1}\right|}{\alpha_{n}}=0 .
$$

For given $x, x_{1} \in C$, let $\left\{x_{n}\right\}$ be a sequence in $C$ generated by the Halpern iteration process:

$$
x_{n+1}=\alpha_{n} x+\left(1-\alpha_{n}\right) T x_{n} \text { for all } n \in \mathbb{N} .
$$

Then $\left\{x_{n}\right\}$ converges strongly to $Q_{F(T)} x$, where $Q_{F(T)}$ is the sunny nonexpansive retraction from $C$ onto $F(T)$.

Note that the nonexpansive mapping $T$ involving in algorithm (5.7) plays an important role in strong convergence of the sequence $\left\{x_{n}\right\}$ defined by (5.7). In our next result, we construct some iterative algorithms using sunny nonexpansive retraction which are independent of such nonexpansive mappings.

Theorem 5.10. Let $X$ be a reflexive Banach space whose norm is uniformly Gâteaux differentiable, $C$ a nonempty closed convex subset of $X$ and $D$ a nonempty subset of $C$. Suppose that $D$ is a sunny nonexpansive retract of $C$. Let $f: C \rightarrow C$ be a weakly contraction mapping with the function $\psi$ and let $Q_{D}$ be the sunny nonexpansive retraction from $C$ onto $D$. Then the sequence $\left\{x_{n}\right\}$ degenerated by the algorithm:

$$
x_{n+1}=Q_{D} f x_{n} \text { for all } n \in \mathbb{N},
$$

converges strongly to $z$, the unique solution of $V I_{D}(C, I-f)$, with the following error estimate:

$$
\left\|x_{n}-z\right\| \leq \Phi^{-1}\left(\Phi\left(\left\|x_{1}-z\right\|\right)\right)-(n-1) .
$$

Proof. Note that $Q_{D} f$ is a weakly contraction mapping from $C$ into itself with the function $\psi$. In fact,

$$
\left\|Q_{D} f x-Q_{D} f y\right\| \leq\|f x-f y\| \leq\|x-y\|-\psi(\|x-y\|) \text { for all } x, y \in C .
$$


Since $Q_{D} f$ is a self-mapping on $C,\left\{x_{n}\right\}$ is well defined. Applying Theorem 2.3 . we obtain that $\left\{x_{n}\right\}$ converges strongly to the unique fixed point $z$ of $Q_{D} f$. The reminder estimate follows from Theorem 2.3 .

Theorem 5.11. Let $X$ be a reflexive Banach space whose norm is uniformly Gâteaux differentiable, $C$ a nonempty closed convex bounded subset of $X$ and $D$ a nonempty subset of $C$ with normal structure. Suppose that $D$ is a sunny nonexpansive retract of $C$. Let $f: C \rightarrow C$ be a nonexpansive mapping and let $Q_{D}$ be the sunny nonexpansive retraction from $C$ onto $D$. Let $x, x_{1}$ be elements in $C$ and let $\left\{\alpha_{n}\right\}$ be a sequence in $[0,1]$ such that

$$
\lim _{n \rightarrow \infty} \alpha_{n}=0, \sum_{n=1}^{\infty} \alpha_{n}=\infty \text { and } \lim _{n \rightarrow \infty} \frac{\left|\alpha_{n}-\alpha_{n-1}\right|}{\alpha_{n}}=0 .
$$

Then the sequence $\left\{x_{n}\right\}$ degenerated by the Helpern iteration process:

$$
x_{n+1}=\alpha_{n} x+\left(1-\alpha_{n}\right) Q_{D} f x_{n} \text { for all } n \in \mathbb{N},
$$

converges strongly to an element of $\Omega_{D}(I-f)$.

Proof. Since $Q_{D} f$ is a nonexpansive mapping from $C$ into itself, hence $F\left(Q_{D} f\right) \neq \emptyset$ and $\left\{x_{n}\right\}$ is well defined. Applying Corollary 5.9, we obtain that $\left\{x_{n}\right\}$ converges strongly to a fixed point $z$ of $Q_{D} f$.

Next, we replace $D$ by $F(T)$, the fixed point of a nonexpansive mapping $T$ : $C \rightarrow C$.

Theorem 5.12. Let $X$ be a reflexive Banach space whose norm is uniformly Gâteaux differentiable norm and $C$ a nonempty closed convex bounded subset of $X$ with normal structure. Let $f, T: C \rightarrow C$ be two nonexpansive mappings. Let $x, x_{1}$ be elements in $C$ and let $\left\{\alpha_{n}\right\}$ be a sequence in [0,1] such that

$$
\lim _{n \rightarrow \infty} \alpha_{n}=0, \sum_{n=1}^{\infty} \alpha_{n}=\infty \text { and } \lim _{n \rightarrow \infty} \frac{\left|\alpha_{n}-\alpha_{n-1}\right|}{\alpha_{n}}=0 .
$$

Then the sequence $\left\{x_{n}\right\}$ degenerated by the Helpern iteration process:

$$
x_{n+1}=\alpha_{n} x+\left(1-\alpha_{n}\right) Q_{F(T)} \text { f } x_{n} \text { for all } n \in \mathbb{N},
$$

converges strongly to $z=P_{F\left(Q_{F(T)} f\right)} x$ which a solution of the variational inequality:

find $\tilde{x} \in F(T)$ such that $\langle(I-f) \tilde{x}, J(\tilde{x}-v)\rangle \leq 0$ for all $v \in F(T)$,

where $P_{F\left(Q_{F(T)} f\right)}$ is the sunny nonexpansive retraction form $C$ onto $F\left(Q_{F(T)} f\right)$.

\section{Applications}

In this section, we give two convergence theorems for finding common fixed points of nonexpansive mappings and common zeros of accretive operators in Banach spaces.

Proposition 6.1. Let $C$ be a nonempty closed convex subset of a strictly convex Banach space $X$ and let $\lambda_{i}>0(i=1,2, \cdots, r)$ such that $\sum_{i=1}^{r} \lambda_{i}=1$. Let $T_{1}, T_{2}, \cdots, T_{r}: C \rightarrow C$ be nonexpansive mappings with $\cap_{i=1}^{r} F\left(T_{i}\right) \neq \emptyset$ and let $T=\sum_{i=1}^{r} \lambda_{i} T_{i}$. Then $T$ is nonexpansive from $C$ into itself and $F(T)=\cap_{i=1}^{r} F\left(T_{i}\right)$. 
Proof. Since $\cap_{i=1}^{r} F\left(T_{i}\right) \subseteq F(T)$ is trivial, we show that $F(T) \subseteq \cap_{i=1}^{r} F\left(T_{i}\right)$. Let $z \in F(T)$ and $v \in \cap_{i=1}^{r} F\left(T_{i}\right)$. Observe that

$$
\begin{aligned}
\|z-v\| & =\left\|\sum_{i=1}^{r} \lambda_{i} T_{i} z-v\right\| \\
& \leq \lambda_{1}\left\|T_{1} z-v\right\|+\lambda_{2}\left\|T_{2} z-v\right\|+\cdots+\lambda_{r}\left\|T_{r} z-v\right\| \\
& \leq \lambda_{1}\|z-v\|+\lambda_{2}\|z-v\|+\cdots+\lambda_{r}\|z-v\|=\|z-v\|,
\end{aligned}
$$

which implies that

$$
\left\|T_{1} z-v\right\|=\left\|T_{2} z-v\right\|=\cdots=\left\|T_{r} z-v\right\|=\|z-v\| .
$$

Since $X$ is a strictly convex, it follows that $T_{1} z=T_{2} z=\cdots=T_{r} z=z$.

Recall that the so-called problem of image recovery is essentially to find a common element of finitely many nonexpansive retracts $C_{1}, C_{2}, \ldots, C_{r}$ of $C$ with $\cap_{i=1}^{r} C_{i} \neq \emptyset$. It is easy to see that every nonexpansive retraction $P_{i}$ of $C$ onto $C_{i}$ is a nonexpansive mapping of $C$ into itself. There is no doubt that the problem of image recovery is equivalent to finding a common fixed point of finitely many nonexpansive mappings $P_{1}, \ldots, P_{r}$ of $\mathrm{C}$ into itself.

Applying Proposition 6.1, we obtain the following result which improves a number of results connected to the problem of image recovery.

Theorem 6.2. Let $X$ be a reflexive strictly convex Banach space whose norm is uniformly Gâteaux differentiable and $C$ a nonempty closed convex subset of $X$. Let $\lambda_{i} \geq 0(i=1,2, \cdots, r)$ such that $\sum_{i=1}^{r} \lambda_{i}=1$ and let $T_{1}, T_{2}, \cdots, T_{r}: C \rightarrow C$ be nonexpansive mappings with $\cap_{i=1}^{r} F\left(T_{i}\right) \neq \emptyset$. Let $\left\{\alpha_{n}\right\}$ be a sequence in [0,1] such that

$$
\lim _{n \rightarrow \infty} \alpha_{n}=0, \sum_{n=1}^{\infty} \alpha_{n}=\infty \text { and } \lim _{n \rightarrow \infty} \frac{\left|\alpha_{n}-\alpha_{n-1}\right|}{\alpha_{n}}=0 .
$$

For given $x, x_{1} \in C$, let $\left\{x_{n}\right\}$ be a sequence in $C$ generated by algorithm:

$$
x_{n+1}=\alpha_{n} x+\left(1-\alpha_{n}\right) \sum_{i=1}^{r} \lambda_{i} T_{i} x_{n} \text { for all } n \in \mathbb{N} .
$$

Then $\left\{x_{n}\right\}$ converges strongly to $Q_{\cap_{i=1}^{r} F\left(T_{i}\right)} x$, where $Q_{\cap_{i=1}^{r} F\left(T_{i}\right)}$ is the sunny nonexpansive retraction from $C$ onto $\cap_{i=1}^{r=1} F\left(T_{i}\right)$.

Proof. Let $T=\sum_{i=1}^{r} \lambda_{i} T_{i}$. Proposition 6.1 implies that $T$ is nonexpansive from $C$ into itself and $F(T)=\cap_{i=1}^{r} F\left(T_{i}\right)$. Hence the result follows from Corollary 5.9.

The following result in an improvement of Theorem 3.3 of Zegeye and Shahzad [26].

Theorem 6.3. Let $X$ be a reflexive strictly convex Banach space with uniformly Gâteaux differentiable norm, $C$ a nonempty closed convex subset of $X$ and $f: C \rightarrow$ $C$ a contraction mapping with Lipschitz constant $k \in[0,1)$. Let $A_{i} \subset X \times X$, $i=1,2, \cdots, r$ be a family of accretive operators with resolvent $J_{t}^{A_{i}}$ for $t>0$ such that $\cap_{i=1}^{r} A_{i}^{-1} 0 \neq \emptyset$ and $\overline{D\left(A_{i}\right)} \subset C \subset \bigcap_{t>0} R\left(I+t A_{i}\right)$. Let $\lambda_{i} \geq 0(i=1,2, \cdots, r)$ 
such that $\sum_{i=1}^{r} \lambda_{i}=1$. For given $x_{1} \in C$, let $\left\{x_{n}\right\}$ be a sequence in $C$ generated by algorithm:

$$
x_{n+1}=\alpha_{n} f x_{n}+\left(1-\alpha_{n}\right) \sum_{i=1}^{r} \lambda_{i} J_{t}^{A_{i}} x_{n} \text { for all } n \in \mathbb{N},
$$

where $\left\{\alpha_{n}\right\}$ is a sequence in $[0,1]$ such that

$$
\lim _{n \rightarrow \infty} \alpha_{n}=0, \sum_{n=1}^{\infty} \alpha_{n}=\infty \text { and } \lim _{n \rightarrow \infty} \frac{\left|\alpha_{n}-\alpha_{n-1}\right|}{\alpha_{n}}=0 .
$$

Then $\left\{x_{n}\right\}$ converges strongly to the unique solution of the variational inequality:

find $\tilde{x} \in \cap_{i=1}^{r} A_{i}^{-1} 0$ such that $\langle(I-f) \tilde{x}, J(\tilde{x}-v)\rangle \leq 0$ for all $v \in \cap_{i=1}^{r} A_{i}^{-1} 0$.

Proof. Note that each $J_{t}^{A_{i}}$ is nonexpansive. Let $T=\sum_{i=1}^{r} \lambda_{i} J_{t}^{A_{i}}$. Proposition 6.1 implies that $T$ is nonexpansive from $C$ into itself and $F(T)=\cap_{i=1}^{r} A_{i}^{-1} 0$. Hence the result follows from Corollary 5.7 .

\section{REFERENCES}

[1] Ya.I. Alber, S. Guerre-Delabriere, Principles of weakly contractive maps in Hilbert spaces, in: I. Gohberg, Yu. Lyubich (Eds.), New Results in Operator Theory, Advances and Appl., vol. 98, Birkhauser, Basel, 1997, 7-22.

[2] T.D. Benavides, G. L. Acedo and H.K.Xu, Iterative solutions for zeros of accretive operators, Math. Nachr., 248-249 (2003), 62-71.

[3] L. Cioranescu, Geometry of Banach spaces, duality mappings and nonlinear problems, Kluwer Aacademic Publishers, 1990.

[4] O. Gäuler, On the convergence of the proximal point algorithm for convex minimization, SIAM J. Control Optim., 29 (1991), 403-419.

[5] K. Goebel and S. Reich, Uniform convexity, hyperbolic geometry, and nonexpansive mappings, Marcel Dekker, Inc., 1984.

[6] K.S. Ha and J.S. Jung, Strong convergence theorems for accretive operators in Banach space, J. Math. Anal. Appl., 147 (2) (1990), 330-339.

[7] B. Halpern, Fixed points of nonexpanding maps, Bull. Amer. Math. Soc., 73 (1967) 957-961.

[8] S. Kamimura and W. Takahashi, Approximating solutions of maximal monotone operators in Hilbert space, J. Approx. Theory, 106 (2000), 226-240.

[9] T.H.Kim and H.K.Xu, Strong convergence of modified Mann iterations, Nonlinear Anal., 61 (2005), $51-60$.

[10] D. Kinderlehrer and G. Stampacchia, An introduction to variational inequalities and their applications, Academic Press, New York, 1980.

[11] W. A. Kirk, A fixed point theorem for mappings which do not increase distances, The Amer. Math. Monthly, 72 (1965), 1004-1006.

[12] J. L. Lions and G. Stampacchia, Variational inequalities, Comm. Pure Appl. Math., 20 (1967), 493-517.

[13] P.E. Mainge, Viscosity methods for zeroes of accretive operators, J. Approx. Theory, 140 (2006), 127- 140

[14] R.H. Martin, Differential equations on closed subsets of a Banach space, Trans. Amer. Math. Soc., 179 (1973), 399-414.

[15] B. Martinet, Regularisation dinequations variationnelles par approximations successives, Rev. FranMcaise Informat. Recherche Operationnelle, 4 (1970), 154-158.

[16] A. Moudafi, Viscosity approximation methods for fixed points problems, J. Math. Anal. Appl., 241 (1) (2000), 46-55.

[17] K. Nakajo, Strong convergence to zeros of accretive operators in Banach spaces, J. Nonlinear Convex Anal., 7 (2006), 71-81.

[18] S. Reich, Strong convergence theorems for resolvents of accretive operators in Banach spaces, J. Math. Anal. Appl., 75 (1980), 287-292. 
[19] B.E. Rhoades, Some theorems on weakly contractive maps, Nonlinear Anal., 47 (2001) 26832693.

[20] R. T. Rockafellar, Monotone operators and the proximal point algorithm, SIAM J. Control Optim., 14 (1976), 877-898.

[21] M. V. Solodov and B. F. Svaiter, Forcing strong convergence of proximal point iterations in a Hilbert space, Math. Programming Ser. A., 87 (2000), 189-202.

[22] W. Takahashi, Nonlinear Functional Analysis, Yokohama 2000.

[23] W. Takahashi and Y. Ueda, On Reich's strong convergence theorems for resolvents of accretive operators, J. Math. Anal. Appl., 104 (1984), 546-553.

[24] H.K. Xu, Viscosity approximation methods for nonexpansive mappings, J. Math. Anal. Appl., 298 (1) (2004), 240-256.

[25] H.K. Xu, Strong convergence of an iterative method for nonexpansive and accretive operators, J. Math. Anal. Appl., 314 (2006), 631-643.

[26] H. Zegeye, N. Shahzad, Strong convergence theorems for a common zero of a finite family of m-accretive mappings, Nonlinear Anal., 66 (2007), 1161-1169.

Department of Applied Mathematics, National Sun Yat-sen University, Kaohsiung 804, TAIWAN

E-mail address: wong@math.nsysu.edu.tw

Department of Applied Mathematics, Shri Shankaracharya College of Engineering and Technology, Junwani, Bhilai - 490020 India

E-mail address: sahudr@yahoo.com

Department of Applied Mathematics, National Sun Yat-Sen University, Kaohsiung 804, TAIWAN

E-mail address: yaojc@math.nsysu.edu.tw 